https://doi.org/10.15407/ujpe65.12.1056

B.I. LEV, A.G. ZAGORODNY

Bogolyubov Institute for Theoretical Physics, Nat. Acad. of Sci. of Ukraine (14 b, Metrolohichna Str., Kyiv,03143,Ukraine; e-mail: blev@bitp.kiev.ua, azagorodny@bitp.kiev.ua)

\title{
STATISTICAL DESCRIPTION OF NON-EQUILIBRIUM MANY-PARTICLE SYSTEMS
}

\begin{abstract}
In most cases, the systems of interacting particles are non-equilibrium. In this review, a new approach based on the application of a non-equilibrium statistical operator is presented, which allows the inhomogeneous distributions of the particles and the temperature to be taken into account. The method uses the saddle-point procedure to find dominant contributions to the partition function of the system and enables all of its thermodynamic parameters to be determined. Probable peculiarities in the behavior of the systems with interaction - such as gravitational systems, systems with Coulombic repulsion, and so forth-under various thermodynamic conditions are predicted. A new approach is proposed to describe non-equilibrium systems in the energy space, which is an extension of the Gibbs approach to macroscopic systems under non-equilibrium conditions. It allows the stationary states and the dynamics of non-equilibrium systems to be described.
\end{abstract}

Ke y w ords: non-equilibrium statistical operator, many-particle systems, stationary states.

\section{Introduction}

Sometimes, systems of interacting particles cannot be described in terms of an ordinary thermodynamic ensemble (for instance, these are self-gravitating systems $[1,2])$, and, as a result, they cannot be considered in the framework of standard methods proposed by the equilibrium statistical mechanics. In particular, if the energy is not an additive quantity, the concept of canonical ensemble is inapplicable for studying the systems with long-range interactions. In such systems, equilibrium states correspond only to local entropy maxima $[3,4]$. In order to determine the equilibrium states for a system of interacting particles and to describe probable phase transitions in it, approaches of two types, statistical and thermodynamic, have been developed. It is generally believed that the mean-field theory is completely acceptable for such systems, although any thermodynamic func-

(C) B.I. LEV, A.G. ZAGORODNY, 2020

1056 tion in this theory depends only on dimensionless combinations of thermodynamic parameters, and the system can be thermodynamically unstable, although the thermodynamic limit does exist [4].

Systems of particles with long-range interactions, such as self-gravitating systems or systems with Coulombic repulsion, do not reach the state of Boltzmann-Gibbs thermodynamic equilibrium. Instead, they turn out in quasistationary states, the lifetime of which can be arbitrarily long, if the number of particles increases. A quantitative description of the instability threshold for the spontaneous symmetry breaking in $d$-dimensional systems was given in work [5]. However, the homogeneous distribution of particles in a system of particles with long-range interactions is unstable. The distribution of particles in such a system is spatially inhomogeneous from the very beginning. The behavior of systems demands different descriptions for different equilibrium ensembles, mainly because any state of a system of particles with long-

ISSN 2071-0194. Ukr. J. Phys. 2020. Vol. 65, No. 12 
range interaction is far from equilibrium, and the time of relaxation to its equilibrium state is very large.

Non-equilibrium stationary states were described in work [6]. Three-dimensional systems can be captured into non-equilibrium quasistationary states thus terminating their evolution toward the thermodynamic equilibrium. As a result, there arises a dilemma: either one uses the postulates of equilibrium statistical mechanics and obtains only the criteria of instability, or one takes possible spatially inhomogeneous particle distributions into account in the framework of other approaches. Inhomogeneous distributions of particles, temperature, and chemical potential can be considered with the help of the nonequilibrium statistical operator [3], which makes allowance for the probability of local changes in the thermodynamic parameters of the system. This approach is based on the fact that the equation of state has to follow from the explicit form of the distribution function for a spatially inhomogeneous system, which can be obtained in the thermodynamic limit $[7,8]$. The system is non-equilibrium a priori, and the inhomogeneity of the particle distribution allows the non-uniform distributions of the temperature, chemical potential, and other thermodynamic parameters to exist.

The formation of a spatially inhomogeneous distribution of interacting particles is a typical problem in the condensed matter physics. The corresponding statistical description has to use a certain procedure to calculate main contributions to the distribution function and has not to bring about the entropy divergence for the infinite system volume. A non-standard method for solving this problem was proposed in works $[9,12]$. This approach uses the HubbardStratonovich representation for the distribution function [13]. It is applied to describe systems of particles with long-range interactions in order to obtain a solution for the particle distribution without putting restrictions on the system volume and the number of particles. It is important that the obtained solution should not possess divergencies in the thermodynamic limit. For this purpose, the saddle-point approximation can be used, in which the number of particles in a limited volume is preserved. The distribution functions in the cases of homogeneous and inhomogeneous particle distributions were obtained in works $[10,11,15]$. However, this approach, makes it possible to determine only the condition for the formation of probable inhomogeneous distributions in the system of particles with long-range interactions.

An important fundamental problem is a search for an adequate approach to the statistical description of self-gravitating systems. The general behavior of such systems has been studied for a long time [16], and this problem has turned out much more complicated than the research of other many-particle systems. In this context, self-gravitating systems are objects that make it possible to verify and to develop the concepts of statistical mechanics and thermodynamics [17]. The statistical description of self-gravitating systems attracts a permanent attention, which is associated with astrophysical problems $[18,28]$ and the formulation of general methods that can be used in other physical situations.

In the case of self-gravitating systems, thermodynamic ensembles are not equivalent. In the framework of the canonical description, there is no heat capacity with a negative value [1], which can be observed for microcanonical ensembles [19]. In the case of microcanonical ensemble, self-destruction corresponds to the "gravithermic catastrophe", but it is associated with the "isothermal collapse" in a canonical ensemble [19]. The self-gravitating system can increase its entropy not only through the growth of its density, because the equilibrium states are associated with the local entropy maximum only. However, if a shortrange repulsive potential is introduced to prevent a complete collapse, the global entropy maximum can be reached now for all achievable energy values. The effective repulsion can be introduced in various ways, because the physical results are rather insensitive to the exact form of regularization. Alternatively, we may consider the classical gas of solid spheres with an introduced excluded volume around every particle [20]. For a gas with purely gravitational interaction between the particles, the corresponding distribution function diverges. Since, in most cases, the states of self-gravitating systems are far from equilibrium, their relaxation time to equilibrium is very large. A homogeneous distribution of identical particles in such a system is unstable, so that the particle distribution is spatially inhomogeneous from the very beginning. The system becomes divided into a set of inhomogeneous clusters that evolve to a more condensed state.

The behavior of a self-gravitating system is described by different methods for different equilibrium 
ensembles. Multiple attempts were made to account for the heterogeneity of particle distributions, but the problem remains yet unsolved. It is so because, if the heterogeneity is taken into consideration, the chemical potential begins to depend on spatial variables. Since the equation of state must couple the temperature and the density, the former, as a thermodynamic parameter, must also depend on spatial coordinates. The standard approach uses a polytropic equation that determines the dependence of the temperature and, hence, the pressure on the concentration. This approach allows one to generate, in particular, stable solutions while considering the gravitational formation of stars. However, it is somewhat inconsistent, because the equation of state must be obtained from the definition of the distribution function, which is unknown for spatially inhomogeneous systems [7, 8]. Therefore, there arises the dilemma mentioned above.

In this review, we present a new approach that is based on the application of the non-equilibrium statistical operator [3], which is better suited to describe systems of particles with long-range interaction. The equations of state and all necessary thermodynamic parameters are governed by the equations that determine the states that provide the largest contribution to the partition function. Therefore, there is no necessity to introduce an additional hypothesis about the temperature dependence of the concentration. This dependence follows from the solution of the corresponding thermodynamic relations that determine the non-equilibrium distribution function. Probable spatially inhomogeneous distributions for the particle concentration and the temperature were obtained in some simple cases. In the case of equilibrium, the well-known result for the distribution function $[21,22]$ was reproduced. It was shown that the proposed approach describes the inhomogeneous particle distribution and allows the required parameters of the system to be determined.

The main idea of the work is to give a detailed description for the system with long-range interaction, which is based on the principles of non-equilibrium statistical mechanics, as well as to obtain probable particle distributions in the case where the particle number and the energy of the system are constant.

Till now, the statistical description of inhomogeneous systems assumed that they are characterized by spatially inhomogeneous distributions of particles, temperature, and chemical potential, as well as by fixed values of the particle number and the energy. In many cases, however, the system heterogeneity is a result of energy changes associated with the external effect of the environment. Therefore, we need to develop a method that would be able to make allowance for the influence of the environment on the behavior of a particular macroscopic system and would allow finding its corresponding states. Fluctuations in the parameters of both the system and the environment must also be taken into account. It was Gibbs who gave us a clue to this approach [53].

In all cases, any macroscopic system interacting with its environment relaxes and finally transits into the equilibrium. The properties of such a system are determined as functions of the environmental characteristics. The equilibrium state for an individual macroscopic system can be achieved under ideal conditions $[21,52,54]$. The influence of the environment finally results in that the thermodynamic parameters of an individual macrosystem coincide with the parameters of the thermostat.

It is known that any state of the system can be described in terms of distribution functions that make it possible to determine all thermodynamic properties of the macroscopic system $[21,52]$. In practice, the statistical description of a macroscopic system requires the knowledge of only a few macroscopic parameters, e.g., the energy. Hence, the principal task consists in developing a method to study the properties of the stationary states in open systems and finding the conditions under which those states do exist. One of the possible ways to solve this general problem could be the application of a description based on the Gibbs approach [53]. The main purpose of our review is to propose a simple way to describe non-equilibrium systems in the energy space [24] and to apply it to the formulation of a new concept of solving the cosmological problem.

\section{Statistical Description of Non-Equilibrium Systems of Interacting Particles}

The statistical thermodynamics of non-equilibrium systems is based on the conservation laws for dynamic variables. When determining the thermodynamic functions of non-equilibrium systems, the representations of the corresponding statistical ensem-

ISSN 2071-0194. Ukr. J. Phys. 2020. Vol. 65, No. 12 
bles involving the non-equilibrium states of those systems are used. We may assume that Gibbs' concept of ensembles can be applied to describe non-equilibrium stationary states of the system as well. In this case, we may define the non-equilibrium ensemble as a set of systems that corresponds to the same external stationary action. Those systems interact with a thermostat in the same manner and can be characterized by all possible values of macroscopic parameters that are allowed by the imposed conditions. In the systems that are situated under identical external stationary conditions, local stationary distributions are formed. If the external conditions depend on the time, the local equilibrium distribution is not stationary. For a nonlocally equilibrium ensemble, the distribution function or the statistical operator of the system [3] has to be determined.

It can also be recalled that the stationary states are only metastable, because they correspond to the local maximum of the entropy. If we assume that the nonequilibrium states of the system can be determined with the help of the inhomogeneous energy distribution $H(\mathbf{r})$ and the inhomogeneous particle concentration $n(\mathbf{r})$, the distribution function for a classical system can be written in the form [3]

$f_{l}=Q_{l}^{-1} \exp \left\{-\int(\beta(\mathbf{r}) H(\mathbf{r})-\eta(\mathbf{r}) n(\mathbf{r})) d \mathbf{r}\right\}$,

where

$Q_{l}=\int D \Gamma \exp \left\{-\int(\beta(\mathbf{r}) H(\mathbf{r})-\eta(\mathbf{r}) n(\mathbf{r})) d \mathbf{r}\right\}$.

The integration in formula (2) is performed over the whole phase space of the system. It should be noted that, in the case of a local equilibrium distribution, the Lagrange multipliers $\beta(\mathbf{r})$ and $\eta(\mathbf{r})$ depend on the spatial coordinates. The microscopic concentration of particles can be presented in the standard form

$n(\mathbf{r})=\sum_{i} \delta\left(\mathbf{r}-\mathbf{r}_{\mathbf{i}}\right)$

A local equilibrium distribution can be introduced, if the time of relaxation in the whole system is larger than the time of relaxation in a local macroscopic region.

After having determined the non-equilibrium statistical operator, we can describe all thermodynamic parameters of the non-equilibrium system. For this purpose, we will generalize the thermodynamic relation for inhomogeneous systems. In order to determine the unknown Lagrange multipliers, let us write the required thermodynamic relation in the form [3]

$-\frac{\delta \ln Q_{l}}{\delta \beta(\mathbf{r})}=\langle H(\mathbf{r})\rangle_{l}, \quad \frac{\delta \ln Q_{l}}{\delta \eta(\mathbf{r})}=\langle n(\mathbf{r})\rangle_{l}$.

These formulas are a natural generalization of the well-known relations for an equilibrium system onto the case of inhomogeneous system. The conservation law for particles and the energy in the system can be written as follows:

$\int n(\mathbf{r}) d \mathbf{r}=N, \quad \int H(\mathbf{r}) d \mathbf{r}=E$.

For the further statistical description of the nonequilibrium system, it is necessary to define the Hamiltonian of the system,

$H=\sum_{i} \frac{p_{i}^{2}}{2 m_{i}}+\frac{1}{2} \sum_{i, j} V\left(\mathbf{r}_{i}, \mathbf{r}_{j}\right)$

where the potential energy of interaction consists of two terms,

$V\left(\mathbf{r}_{i}, \mathbf{r}_{j}\right)=-W\left(\mathbf{r}_{i}, \mathbf{r}_{j}\right)+U\left(\mathbf{r}_{i}, \mathbf{r}_{j}\right)$,

Namely, $W\left(\mathbf{r}_{i} \mathbf{r}_{j}\right)$ and $U\left(\mathbf{r}_{i} \mathbf{r}_{j}\right)$ describe the attraction and repulsion, respectively. Then the energy density looks like

$H(\mathbf{r})=\frac{p^{2}(\mathbf{r})}{2 m(\mathbf{r})} n(\mathbf{r})-\frac{1}{2} \int W\left(\mathbf{r}, \mathbf{r}^{\prime}\right) n(\mathbf{r}) n\left(\mathbf{r}^{\prime}\right) d \mathbf{r}^{\prime}+$

$+\frac{1}{2} \int U\left(\mathbf{r}, \mathbf{r}^{\prime}\right) n(\mathbf{r}) n\left(\mathbf{r}^{\prime}\right) d \mathbf{r}^{\prime}$

This representation can be used, if we divide the whole space into equal regions with the same mass and consider their motion in the phase space as the flow of an incompressible fluid. For a system of interacting particles, the non-equilibrium statistical operator can be written in the form

$Q_{l}=\int D \Gamma \exp [S(\mathbf{r})] Q_{\text {int }}$,

where

$S(\mathbf{r})=-\int\left[\beta(\mathbf{r}) \frac{p^{2}(\mathbf{r})}{2 m(\mathbf{r})}-\eta(\mathbf{r})\right] n(\mathbf{r}) d \mathbf{r}$, 
$Q_{\mathrm{int}}=\exp \left\{-\frac{1}{2} \int \beta(\mathbf{r}) V\left(\mathbf{r}, \mathbf{r}^{\prime}\right) n(\mathbf{r}) n\left(\mathbf{r}^{\prime}\right) d \mathbf{r} d \mathbf{r}^{\prime}\right\}$.

The integration over the phase space means

$D \Gamma=\frac{1}{(2 \pi \hbar)^{3}} \prod_{i} d r_{i} d p_{i}$.

In order to formally integrate in the second part of this representation, additional field variables can be introduced with the use of the theory of Gaussian integrals $[12,13]$ :

$\exp \left\{-\frac{\nu^{2}}{2} \int \beta \omega n n^{\prime} d r d r^{\prime}\right\}=$

$=\int D \sigma \exp \left\{-\frac{\nu^{2}}{2} \int(\beta \omega)^{-1} \sigma \sigma^{\prime} d r d r^{\prime}-\nu \int \sqrt{\beta} \sigma n d r\right\}$.

Here,

$D \sigma=\frac{\prod_{s} d \sigma_{s}}{\sqrt{\operatorname{det} 2 \pi \beta \omega\left(\mathbf{r}, \mathbf{r}^{\prime}\right)}}$

and $\omega^{-1}\left(\mathbf{r}, \mathbf{r}^{\prime}\right)$ is an inverse operator that satisfies the condition $\omega^{-1}\left(\mathbf{r}, \mathbf{r}^{\prime}\right) \omega\left(\mathbf{r}^{\prime}, \mathbf{r}^{\prime \prime}\right)=\delta\left(\mathbf{r}-\mathbf{r}^{\prime \prime}\right)$. The interaction energy is Green's function for this operator, and $\nu^{2}= \pm 1$ depending on the sign of the interaction or potential energy. After the proposed transformation, the field of the variable $\sigma(\mathbf{r})$ contains the same information as the initial distribution function does, i.e. all information about possible spatial states of the system. Now, the statistical operator can be rewritten in the form

$Q_{l}=\int D \Gamma D \varphi D \psi \times$

$\times \exp \left\{-\int s(\mathbf{r}) n(\mathbf{r}) d \mathbf{r}-\int v\left(\mathbf{r}, \mathbf{r}^{\prime}\right) d \mathbf{r} d \mathbf{r}^{\prime}\right\}$,

where

$s(\mathbf{r})=\beta(\mathbf{r}) \frac{p^{2}(\mathbf{r})}{2 m(\mathbf{r})}+\eta(\mathbf{r})+\sqrt{\beta(\mathbf{r}))} \psi(\mathbf{r})+i \sqrt{\beta(\mathbf{r}))} \varphi(\mathbf{r})$,

and the part that originates from the interaction equals

$$
\begin{aligned}
& v\left(\mathbf{r}, \mathbf{r}^{\prime}\right)=\frac{1}{2}\left[\beta(\mathbf{r}) W\left(\mathbf{r}, \mathbf{r}^{\prime}\right)\right]^{-1} \psi(\mathbf{r}) \psi\left(\mathbf{r}^{\prime}\right)- \\
& -\frac{1}{2}\left[\beta(\mathbf{r}) U\left(\mathbf{r}, \mathbf{r}^{\prime}\right)\right]^{-1} \varphi(\mathbf{r}) \varphi\left(\mathbf{r}^{\prime}\right) .
\end{aligned}
$$

In the general functional integral, the integration can be carried out over the phase space, if we use the definition of particle concentration and sum up over the occupation numbers. The mathematical transformations for this functional can be found in original works [37-40]. Afterward, the non-equilibrium statistical operator can be rewritten in the form

$Q_{l}=\int D \varphi D \psi d \xi \exp \{-S(\varphi(\mathbf{r}), \psi(\mathbf{r}), \xi(\mathbf{r}), \beta(\mathbf{r}))\}$,

where the effective non-equilibrium "local thermodynamic potential" reads

$$
\begin{aligned}
& S=-\frac{1}{2} \int\left[\beta(\mathbf{r}) W\left(\mathbf{r}, \mathbf{r}^{\prime}\right)\right]^{-1} \psi(\mathbf{r}) \psi\left(\mathbf{r}^{\prime}\right)- \\
& -\frac{1}{2} \int\left[\beta(\mathbf{r}) U\left(\mathbf{r}, \mathbf{r}^{\prime}\right)\right]^{-1} \varphi(\mathbf{r}) \varphi\left(\mathbf{r}^{\prime}\right) d \mathbf{r} d \mathbf{r}^{\prime}- \\
& -\int\left[\xi(\mathbf{r})\left(\frac{2 \pi m(\mathbf{r})}{\hbar^{3} \beta(\mathbf{r})}\right)^{3 / 2} \times\right. \\
& \times \exp \sqrt{\beta(r)} \psi(\mathbf{r}) \cos (\sqrt{\beta(\mathbf{r}))} \varphi(\mathbf{r})] d \mathbf{r}
\end{aligned}
$$

Here, the introduced new variable $\xi(\mathbf{r}) \equiv \exp \eta(\mathbf{r})$ can be interpreted as the chemical activity. The statistical operator in the presented form makes it possible to apply effective methods developed in the quantum field theory, but imposing no additional integration restrictions on the field variables and using no perturbation theory.

The functional $S(\varphi(\mathbf{r}), \xi(\mathbf{r}), \beta(r))$ depends on the distributions of field variables, $\varphi(\mathbf{r})$, chemical activity, $\xi(\mathbf{r})$, and inverse temperature, $\beta(\mathbf{r})$. Now, we can use the saddle-point method to find the asymptotic value of the statistical operator $Q_{l}$, if the number of particles $N$ tends to infinity $(N \rightarrow \infty)$. The dominant contributions are made by the states that satisfy the conditions of functional extremum. It is evident that the saddle-point equations are thermodynamic relations, and they can be written as equations for the field variable,

$\frac{\delta S}{\delta \varphi(\mathbf{r})}=0, \quad \frac{\delta S}{\delta \psi(\mathbf{r})}=0$,

provided that the number of particles is constant,

$\left.\frac{\delta S}{\delta(\eta(\mathbf{r}))}=-\int \frac{\delta S}{\delta(\xi(\mathbf{r}))} \xi(\mathbf{r})\right) d \mathbf{r}=N$,

ISSN 2071-0194. Ukr. J. Phys. 2020. Vol. 65, No. 12 
and the energy of the system is conserved,

$\left.-\int \frac{\delta S}{\delta(\beta(\mathbf{r}))} \xi(\mathbf{r})\right) d \mathbf{r}=E$

The solutions of the equations obtained in such a way completely determine all thermodynamic parameters and describe the general behavior of the system of interacting particles irrespective of whether the corresponding particle distribution is spatially inhomogeneous or not. In principle, the equations presented above describe a multiparticle problem in the thermodynamic limit. A spatially inhomogeneous solution of those equations corresponds to the distribution of interacting particles. Such heterogeneous behavior is related to the nature and intensity of interaction. In other words, the accumulation of particles in a finite spatial region (the formation of inhomogeneity) reflects the spatial distribution of fields, activity, and temperature. It is very important to note that this is the approach where the non-uniform distribution of the temperature, which may depend on the spatial distribution of particles in the system, can be taken into account.

\section{Systems of Particles with Long-Range Interaction}

The definition of $Q_{l}$ contains the inverse interaction energy operator. Therefore, for the further description, we have to determine this operator. Let us firstly consider a system with attractive gravitational interaction. In the general case of long-range interactions, such as the Coulombic interaction or Newtonian gravitational one, the inverse operator in the continuous limit can be given by the formula

$U^{-1}\left(\mathbf{r}, \mathbf{r}^{\prime}\right)=-\frac{1}{4 \pi G m^{2}} \triangle_{\mathbf{r}} \delta\left(\mathbf{r}-\mathbf{r}^{\prime}\right)=L_{\mathbf{r} \mathbf{r}^{\prime}}^{\psi} \delta\left(\mathbf{r}-\mathbf{r}^{\prime}\right)$

where $\Delta_{\mathrm{r}}$ is the Laplace operator in the real space. The number of realistic interactions for which the inverse operator can be found is confined. Difficulties in obtaining the inverse operator can be avoided by introducing a collective variable that corresponds to the relation between the introduced fields at the saddle-point trajectories. It is known from the cosmology that two masses move away from each other at the velocity $v=H\left|\mathbf{r}-\mathbf{r}^{\prime}\right|$, where $H$ is the Hubble constant, and $\left|\mathbf{r}-\mathbf{r}^{\prime}\right|$ is the distance between them. The kinetic energy of the relative motion of each mass can be written in the form

$T=\frac{m(\mathbf{r})}{2} H^{2}\left(\mathbf{r}-\mathbf{r}^{\prime}\right)^{2}$

and transformed into the energy of interaction between two masses located at different spatial points,

$\left.W(\mathbf{r})-\mathbf{r}^{\prime}\right)=\frac{m(\mathbf{r})}{2} H^{2}\left(\mathbf{r}-\mathbf{r}^{\prime}\right)^{2}+\frac{m\left(\mathbf{r}^{\prime}\right)}{2} H^{2}\left(\mathbf{r}-\mathbf{r}^{\prime}\right)^{2}$,

For a homogeneous mass distribution, this formula can be rewritten in the form $W\left(\mathbf{r}-\mathbf{r}^{\prime}\right)=m(\mathbf{r}) H^{2}(\mathbf{r}-$ $\left.-\mathbf{r}^{\prime}\right)^{2}$. Then, the inverse operator looks like

$L_{\mathbf{r \mathbf { r } ^ { \prime }}}^{\varphi}=\frac{1}{m H^{2}} \frac{d^{2}}{d r^{2}}$

In our case (long-range attraction and even longerrange repulsion between the particles), let us rewrite the non-equilibrium statistical operator in the form

$Q_{l}=\int D \varphi D \psi d \xi \exp \{-S(\varphi(\mathbf{r}), \psi(\mathbf{r}), \xi(\mathbf{r}), \beta(\mathbf{r}))\}$

where the effective non-equilibrium "local thermodynamic potential" reads

$S(\varphi(\mathbf{r}), \psi(\mathbf{r}), \xi(\mathbf{r}), \beta(\mathbf{r}))=$

$=\int \frac{1}{2 r_{\varphi}} \varphi(\mathbf{r}) L_{\mathbf{r r}^{\prime}}^{\varphi} \varphi\left(\mathbf{r}^{\prime}\right)+\frac{1}{2 r_{\psi}} \psi(\mathbf{r}) L_{\mathbf{r r}^{\prime}}^{\psi} \psi\left(\mathbf{r}^{\prime}\right)+$

$+\xi(\mathbf{r}) \Lambda^{-3} \exp \sqrt{\beta(r)} \psi(\mathbf{r}) \cos [\sqrt{\beta(\mathbf{r})} \varphi(\mathbf{r})] d \mathbf{r}$.

Here, the quantities $\beta, \varphi$, and $\psi$ depend on the spatial coordinate. In this representation, we used the de Broglie thermal wavelength

$\Lambda(\mathbf{r})=\left(\frac{2 m(\mathbf{r})}{\hbar^{2} \beta(\mathbf{r})}\right)^{1 / 2}$

and defined the interaction lengths

$r_{\psi}(\mathbf{r})=4 \pi G m^{2} \beta(r)$,

$r_{\varphi}(\mathbf{r})=\frac{2}{m H^{2}}$.

Now, knowing the representation for the distribution function, we can describe various real systems and determine the thermodynamic parameters for a nonequilibrium system. 


\section{System of Particles with Repulsive Interaction}

First of all, let us consider a system characterized by only a repulsive interaction between the particles. In this case, $\psi=0$, and we can express the "local thermodynamic potential" in the simple form

$$
\begin{aligned}
& S=\int\left\{\frac{1}{2} \varphi(\mathbf{r}) L_{\mathbf{r r}^{\prime}} \varphi\left(\mathbf{r}^{\prime}\right)+\right. \\
& \left.+\xi(\mathbf{r}) \Lambda^{-3}(\mathbf{r}) \cos [\sqrt{\beta(\mathbf{r})} \varphi(\mathbf{r})]\right\} d \mathbf{r} .
\end{aligned}
$$

The functional $S(\varphi(\mathbf{r}), \xi(\mathbf{r}), \beta(r))$ depends on the field, chemical activity, and inverse temperature distributions. In order to find the asymptotic value of the statistical operator $Q_{l}$, we can use the saddlepoint method, if the number of particles $N$ tends to infinity. In this case, the dominant contributions are made by the states that satisfy the extremum condition for the functional. It can be shown that the saddle-point equation is a thermodynamic relation that can be reduced to the equation for the field variable,

$$
\frac{\delta S}{\delta \varphi(\mathbf{r})}=0
$$

the condition that the number of particles is constant,

$$
\frac{\delta S}{\delta(\eta(\mathbf{r}))}=\int \frac{\delta S}{\delta(\xi(\mathbf{r}))} \xi(\mathbf{r}) d \mathbf{r}=N
$$

and the conservation law for the energy of the system,

$\int \frac{\delta S}{\delta \beta(\mathbf{r})} \xi(\mathbf{r}) d \mathbf{r}=E$.

The solutions of the equations given above completely determine all thermodynamic functions and describe the general behavior of interacting systems with spatially homogeneous or inhomogeneous particle distributions. The indicated system of equations solves, in principle, the many-particle problem in the thermodynamic limit. It is very important to note that only this approach allows one to consider the inhomogeneous temperature distribution that may depend on the spatial distribution of particles in the system.

In order to obtain more information about the behavior of interacting systems, let us introduce some new variables. From the constancy condition for the particle number, $\int \rho(\mathbf{r}) d \mathbf{r}=N$, let us introduce the macroscopic density function

$\rho(\mathbf{r}) \equiv \Lambda^{-3}(\mathbf{r}) \xi(\mathbf{r}) \cos (\sqrt{\beta(\mathbf{r})} \varphi(\mathbf{r}))$.

In the absence of an interaction (free particles), $\varphi(\mathbf{r})=0$. Then, if we express the chemical activity in terms of the chemical potential, $\xi(\mathbf{r})=$ $=\exp (\mu(\mathbf{r}) \beta(\mathbf{r}))$, we obtain the known relation $\beta(\mathbf{r}) \mu(\mathbf{r})=\ln \rho(\mathbf{r}) \Lambda^{3}(\mathbf{r})$, which generalizes the relation from the equilibrium statistical mechanics [21]. Now, the equation for energy conservation takes the form

$\frac{1}{2} \int \frac{\rho(\mathbf{r})}{\beta(\mathbf{r})}\{3-\sqrt{\beta(r)} \varphi(\mathbf{r}) \tan [\sqrt{\beta(\mathbf{r})} \varphi(\mathbf{r})]\} d \mathbf{r}=E$

From whence, we obtain the relation for the chemical potential,

$$
\begin{aligned}
& \frac{\delta E}{\delta V} \frac{\delta V}{\delta N}=\frac{1}{2} \frac{\rho(\mathbf{r})}{\beta(\mathbf{r})} \times \\
& \times\{3-\sqrt{\beta(r)} \varphi(\mathbf{r}) \tan [\sqrt{\beta(\mathbf{r})} \varphi(\mathbf{r})]\}=\mu(\mathbf{r}) \rho(\mathbf{r}),
\end{aligned}
$$

which results in the following formula for the chemical potential:

$\mu(\mathbf{r}) \beta(\mathbf{r})=\frac{3}{2}-\frac{1}{2} \sqrt{\beta(r)} \varphi(\mathbf{r}) \tan [\sqrt{\beta(\mathbf{r})} \varphi(\mathbf{r})]$.

The same approach also brings us to the equation of state of the type

$P=\frac{1}{\beta} \frac{\delta S}{\delta V}$

The corresponding local equation of state looks like

$P(\mathbf{r}) \beta(\mathbf{r})=\rho(\mathbf{r})\left[\mu(\mathbf{r}) \beta(\mathbf{r})-\frac{1}{2}\right]$.

For the ideal gas, we obtain the known equation of state, because $\varphi(\mathbf{r})=0$ and $P \beta=\rho$. In this case, we obtain $\mu(\mathbf{r}) \beta(\mathbf{r})=3 / 2$, and the equation of state reproduces the equation of state for the ideal gas. The energy of the system equals $E=\frac{3}{2} N k T$. This formula corresponds to the well-known results [21]. On the basis of Eq. (32), a conclusion can be drawn that the condition $\mu(\mathbf{r}) \beta(\mathbf{r})<1 / 2$ corresponds to the appearance of a negative pressure, $P(\mathbf{r}) \beta(\mathbf{r})<0$, and this result satisfies the necessary condition of vacuum in the

ISSN 2071-0194. Ukr. J. Phys. 2020. Vol. 65, No. 12 
general theory of relativity. This condition is fulfilled in the realistic state $\sqrt{\beta(r)} \varphi(\mathbf{r}) \tan [\sqrt{\beta(\mathbf{r})} \varphi(\mathbf{r})]<2$ for the constant temperature and for the total energy $E<\frac{1}{2} N k T$. This assumes that the energy of every particle is lower than the thermal energy. In this specific case, the energy of the system is lower than the total thermal energy of the particles. This very specific condition can be associated with particular properties of the vacuum, If the definition of the chemical potential is taken into account, we can rewrite the density in the form

$\rho(\mathbf{r}) \equiv \Lambda_{e}^{-3}(\mathbf{r}) \exp \left[\frac{1}{2} \sigma(\mathbf{r}) \tan \sigma(\mathbf{r})\right] \cos \sigma(\mathbf{r})$,

where a new variable $\sigma=\sqrt{\beta(r)} \varphi(\mathbf{r})$ was introduced, and the de Broglie wavelength is redefined:

$\Lambda_{e}=\left[\frac{\hbar^{2} \beta(\mathbf{r}) e}{2 m(\mathbf{r})}\right]^{1 / 2}$.

The local thermodynamic potential can be rewritten in the form

$$
\begin{aligned}
& S=\int\left\{\frac{1}{2} \frac{\sigma(\mathbf{r})}{\sqrt{\beta(r)}} L_{\mathbf{r r}^{\prime}} \frac{\sigma\left(\mathbf{r}^{\prime}\right)}{\sqrt{\beta(r)}}+\right. \\
& \left.+\Lambda_{e}^{-3}(\mathbf{r}) \exp \left[\frac{1}{2} \sigma(\mathbf{r}) \tan \sigma(\mathbf{r})\right] \cos \sigma(\mathbf{r})\right\} d \mathbf{r} .
\end{aligned}
$$

If the temperature and the mass of particles are constant, the local entropy can be determined in the mean-field approximation:

$$
\begin{aligned}
& S=\int\left\{\frac{1}{2 \beta} \sigma(\mathbf{r}) L_{\mathbf{r} \mathbf{r}^{\prime}} \sigma\left(\mathbf{r}^{\prime}\right)+\right. \\
& \left.+\Lambda_{e}^{-3} \exp \left[-\frac{1}{2} \sigma(\mathbf{r}) \tan \sigma(\mathbf{r})\right] \cos \sigma(\mathbf{r})\right\} d \mathbf{r} .
\end{aligned}
$$

Now, the equation for the field variable can be rewritten in the form

$$
L_{\mathbf{r r}^{\prime}} \sigma\left(\mathbf{r}^{\prime}\right)-\beta \frac{d V(\sigma)}{d \sigma}=0
$$

where the potential energy

$V=\Lambda_{e}^{-3} \exp \left[-\frac{1}{2} \sigma(\mathbf{r}) \tan \sigma(\mathbf{r})\right] \cos \sigma(\mathbf{r})$

is a function of the field variable. This potential has a minimum, if $3 \sin 2 \sigma=-2 \sigma$. At small $\sigma$-values, there exist two different solutions, $\sigma=0$ and $\sigma^{2}=1$, the effective potential takes a very simple form, $V(\sigma)=$ $=\left(1-\sigma^{2}\right)$, and the equation for the field variable reads: $L_{\mathbf{r r}^{\prime}} \sigma\left(\mathbf{r}^{\prime}\right)+2 \beta \psi=0$. In the general case, the potential energy of the field has an oscillatory character with a decreasing amplitude. Then one can analyze the possible spatial solution for the field variable and the behavior of this field in the time.

If the inverse operator is determined, we can find the spatial dependence of the fundamental scalar field by solving the equation

$\frac{1}{m H^{2}} \frac{d^{2} \sigma}{d r^{2}}-\beta \frac{d V(\sigma)}{d \sigma}=0$

At small $\sigma$, it transforms into the equation

$\frac{d^{2} \sigma}{d r^{2}}+2 \beta m H^{2} \sigma=0$

which has the periodic solution $\sigma=\cos (\sqrt{2 m \beta} H r)$ with the spatial period equal to $2 \pi /(\sqrt{2 m \beta} H)$. At distances shorter than this value, the fundamental scalar field can be assumed constant in the space, but it can change in the time. To determine the evolution of such a field, we can formulate a dynamic equation.

In particular, this solution can describe the formation of a bubble of a new phase in the inflation theory of the Universe [42-44], whereas the introduced field variable plays the role of the fundamental scalar field and makes allowance for a repulsive interaction in the considered system. In this sense, the Ginzburg-Landau equation for the fundamental scalar field written in the standard form can be used as a dynamic field equation,

$\frac{\partial \sigma(\mathbf{r}, t)}{\partial t}=-\gamma \frac{\delta S}{\delta \sigma(\mathbf{r})}=-\gamma \frac{d V(\sigma)}{d \sigma}$

where $\gamma$ is the dynamic viscosity coefficient [50]. This is an evolutionary equation, which is virtually applicable to a number of systems with the nonconservative order parameter. We may assume that the dynamics of the Universe is governed by the entropy growth. The evolution in the non-equilibrium state will be determined under the influence of the entropy landscape and the morphological instability of the parameters. The dynamics of the system is dissipative, which can lead to a local reduction of the entropy. 


\section{System with Coulombic-Type Interaction}

Systems of particles with Coulombic interaction (Coulombic-like systems) - such as plasma, colloidal systems, electrolyte solutions, electron gas in solids, and so forth - are widely spread both in the Nature and under the laboratory conditions. Soft matter of a lot of types - such as surfactant solutions, colloids in various solvents, and dust particles in plasma demonstrates the self-organization. One of the most important problems in this case is the statistical description of Coulombic systems with high concentrations of interacting particles. As the particle concentration increases, the formation of various crystal structures, transitions between crystal phases with different symmetries, or melting take place. The theoretical description of such systems is rather complicated, because the standard methods of statistical mechanics cannot be applied to inhomogeneous systems with the Coulombic interaction. So, there emerges the necessity to use methods that would account for the contribution of dominant configurations to the distribution function and, simultaneously, would not lead to divergences in the free energy, when the system volume infinitely increases. The purpose of this review consists in applying the quantummechanical field approach to both the statistical description of a Coulombic system and the calculation of the thermodynamic characteristics for the homogeneous or inhomogeneous distribution of interacting particles, as well as to studying the space-periodic condensed structures. Below, we will consider electrically neutral systems. Either the Coulombic interaction potential or the effective screened potential will be used depending on the applied model. In the case of a Coulombic system that is neutral in whole, the inverse operator for the screened interaction in the limiting continuum case should be considered in the operator sense, i.e.

$U^{-1}\left(\mathbf{r}, \mathbf{r}^{\prime}\right)=-\frac{1}{4 \pi G Q^{2}}\left(\Delta_{\mathbf{r}}-\varkappa^{2}(\mathbf{r})\right) \delta\left(\mathbf{r}-\mathbf{r}^{\prime}\right)=$

$=L_{\mathbf{r r}^{\prime}}^{\psi} \delta\left(\mathbf{r}-\mathbf{r}^{\prime}\right)$,

where, as before, $\Delta_{\mathbf{r}}$ is the Laplace operator in the real space, and $\varkappa(\mathbf{r})$ is the inverse screening length. In our case, the relation

$$
(\nabla \varphi(\mathbf{r}))^{2}=\nabla(\varphi(\mathbf{r}) \nabla \varphi(\mathbf{r}))-\varphi(\mathbf{r}) \Delta \varphi(\mathbf{r})
$$

can be used to determine the "local thermodynamic potential". When integrating it, the integral of the first term can be represented as a surface integral with $\varphi(\mathbf{r})=0$ at the surface. Then the local entropy can be represented in the form

$S=\int d \mathbf{r}\left\{\frac{1}{2 r_{e}}\left[(\nabla \varphi(\mathbf{r}))^{2}+\varkappa^{2} \varphi^{2} \mathbf{r}\right]-\right.$

$\left.-\xi(\mathbf{r}) \Lambda^{-3}(\mathbf{r}) \cos \varphi(\mathbf{r})\right\}$,

where $r_{e}=4 \pi Q^{2} \beta$, if the temperature is constant.

Let us consider a one-dimensional system with a linear particle concentration. A charge distributed along a macromolecule can be regarded as an example of such a system. Let us consider a cylindrical molecule with length $L$ and radius $r \ll L$. Let the Coulombic charges be located on the cylinder axis. In this case, the problem can be solved exactly. In particular, the local thermodynamic potential of a system of charged particles in the one-dimensional case can be represented as follows:

$S=\frac{V}{L} \int_{0}^{L} d z\left\{\frac{1}{r_{e}}\left(\frac{d \varphi}{d z}\right)^{2}-\xi \Lambda^{-3} \cos \varphi\right\}$,

and the equation for the saddle-point field looks like the sine-Gordon equation,

$\frac{1}{r_{e}}\left(\frac{d^{2} \varphi}{d z^{2}}\right)+\xi \Lambda^{-3} \sin \varphi=0$.

The field equation has the first integral

$\frac{1}{r_{e}}\left(\frac{d \varphi}{d z}\right)^{2}+\xi \Lambda^{-3} \cos \varphi=C$

and the exact solution

$\varphi=4 \arctan \exp \left(z \sqrt{r_{e} \xi \Lambda^{-3}}\right)$.

Substituting this solution into the expression for the local entropy, we obtain

$S=2 \xi \Lambda^{-3} V\left\{\frac{2 E(p)}{p^{2} K(p)}-\frac{1}{p^{2}}+1\right\}-\xi \Lambda^{-3} V$,

where

$p=\sqrt{\frac{2 \xi \Lambda^{-3}}{C+\xi \Lambda^{-3}}}$.

ISSN 2071-0194. Ukr. J. Phys. 2020. Vol. 65, No. 12 
Finally, by applying the normalization condition, we obtain the following exact expression for the local entropy:

$S=S_{B}+8\left(N \frac{L}{r_{e}}\right)^{1 / 2}$.

So, the local entropy of interacting particles increases with the particle number and/or the size of the system.

The spatial boundary conditions may be responsible for the appearance of a periodic structure. The period of the structure equals $l=L\left(\frac{L}{N) r_{e}}\right)^{1 / 2}$ and increases, as the particle number decreases. Such a system is homogeneous on the macroscopic scale, but the particles can be periodically distributed in space.

In the case of a two-dimensional system, the exact solution can be obtained for a uniform particle distribution. Let us consider the Coulombic-like potential

$\omega_{i j}=\frac{Q^{2}}{\langle r\rangle} \ln \varkappa r_{i j}$,

where $r_{i j}$ is the distance between the particles, and $\langle r\rangle$ is its average value. This model potential has the same form as the interaction potential between two uniformly charged lines in the three-dimensional space. Hence, the motion of real charges in a twodimensional plane is similar to the motion of parallel lines oriented perpendicularly to the plane of a twodimensional system. In the continuous limit, the corresponding partition function can be written in the standard form:

$Z_{N}=\int \exp [-\beta H(r, p)] d^{N} \mathbf{r} d^{N} \mathbf{p}$

where the Hamiltonian equals

$H(r, p)=\sum_{i} \frac{p_{i}^{2}}{2 m}+\frac{1}{2} \sum_{i, j} \omega_{i j}$.

In the two-dimensional case, the equation of state can be obtained from the relation

$P=k T \frac{\partial \ln Z_{n}}{\partial S}$

where $S$ is the squared circle radius $R$. By introducing the dimensionless variable $r_{i}=r_{i} / S^{1 / 2}$, the partition function can be rewritten in the form

$Z_{N}=S^{N} \int \exp [-\beta H(r, p)] d^{N} \mathbf{r}^{\prime} d^{N} \mathbf{p}^{\prime}, r_{i}^{\prime}=\frac{r_{i}}{S^{1 / 2}}$.
Then the derivative of the partition function with respect to the "volume" can be represented as follows:

$$
\begin{aligned}
& \frac{\partial Z_{N}}{\partial S}=\frac{N Z}{S}-\frac{S^{N}}{k T} \times \\
& \times \int \exp (-\beta H) \frac{1}{2} \sum_{i, j} \frac{r_{i j}}{2 S} \frac{\partial \omega_{i j}}{\partial r_{i j}} d^{N} \mathbf{r}^{\prime} d^{N} \mathbf{p}^{\prime}
\end{aligned}
$$

or

$\frac{\partial Z_{N}}{\partial S}=\frac{N Z}{S}-\frac{N(N-1) Z}{4 S k T}$.

Substituting this result into the equation of state, we obtain the following exact solution for the twodimensional Coulombic system:

$P S=N k T\left\{1+\frac{(N-1) Q^{2}}{4 k T\langle r\rangle}\right\}$.

If we take into account that the average distance between the particles, $\langle r\rangle$, is proportional to $n^{-1 / 2}$, where $n$ is the surface concentration of particles, we can show that, in the case of high concentration, the system of interacting particles becomes unstable, so that an inhomogeneous particle distribution may arise. The inhomogeneous distributions of this kind are a result of the long-range character of the Coulombic interaction. In the case of intense interaction, the Coulombic-like system is unstable in general, so the minimum value of the thermodynamic potential is reached in the case where the particle distribution is inhomogeneous.

Let us apply the proposed approach to find the states associated with Wigner crystals. In the twodimensional case, the effective thermodynamic potential can be written as follows:

$S=\frac{V}{S} \int d x d y\left\{\frac{1}{r_{e}}\left((\nabla \varphi)^{2}+\varkappa^{2} \varphi^{2}\right)-\xi \Lambda^{-3} \cos \varphi\right\}$,

where $V / S=h$ is the thickness of the twodimensional layer. In the general case, the equation for saddle-point states takes the form

$\frac{1}{r_{e}}\left\{\Delta_{2} \varphi-\varkappa^{2} \varphi\right\}+\xi \Lambda^{-3} \sin \varphi=0$,

where $\Delta_{2}$ is the Laplace operator in the twodimensional case. Thus, we obtain the first integral in the form

$\frac{1}{r_{e}}\left[(\nabla \varphi)^{2}-\varkappa^{2} \varphi^{2}\right]+\xi \Lambda^{-3} \cos \varphi=E$, 
where $E$ is an integration constant, which is to be determined from the condition that a solution for the field does exist. Although this equation cannot be solved in the general case, it is a tool to study numerous Coulombic systems with interaction under various external conditions. In particular, the chemical activity can be obtained from the normalization condition $(V / S) \int d x d y \xi \Lambda^{-3} \cos \varphi=N$. In terms of the density function $\rho$, the first integral can also be written in the form

$S=\frac{V}{S} \int d x d y\left\{E+2 \frac{\varkappa^{2}}{r_{e}} \arccos ^{2}\left(\frac{\rho}{\xi \Lambda^{-3}}\right)-2 \rho\right\}$,

where the value of the integral over the coordinate space is evaluated in terms of the averaged concentration.

From the minimization condition for the effective local entropy, we draw a conclusion that the solution exists, if $\xi \Lambda^{-3}=\rho_{0}$. Then the effective local entropy takes the form

$S \simeq S_{B}+N\left\{\frac{\pi^{2} \varkappa^{2}}{4 n r_{e}}-1\right\}$.

Let us use the approach proposed above to describe a stationary system of dust particles in weakly ionized plasma. In this case, the method again allows us to determine the states that give the dominant contribution to the distribution function, i.e. this is the saddle-point approximation. In the general case, the equation for saddle points looks like

$\frac{1}{r_{e}}\left\{\Delta \varphi-\varkappa^{2} \varphi\right\}+\xi \Lambda^{-3} \sin \varphi=0$.

The difficulty consists in that we do not know a three-dimensional solution of the sine-Gordon equation, which would determine the field variable under the normalization condition

$\int d V \xi \Lambda^{-3} \cos \varphi=N$

It should be noted that this condition makes it possible to introduce the concentration of particles as $\rho(\mathbf{r})=\xi \Lambda^{-3} \cos \varphi(\mathbf{r})$. From whence, it follows that the first integral of the equation can be written in the form

$\frac{1}{r_{e}}\left\{(\nabla \varphi)^{2}+\varkappa^{2} \varphi^{2}\right\}+\xi A \cos \varphi=E$,

1066 where $E$ is an integration constant. Similarly to the two-dimensional case, this equation cannot be solved explicitly.

Like the two-dimensional Coulombic system, the examined system becomes unstable as a whole in the case of intense interaction, so the minimum value of the free energy is achieved at an inhomogeneous particle distribution. Let us determine the states associated with the formation of Wigner crystals. Taking into account that the distribution density is always positive and assuming that the Wigner crystal does exist, let us select the periodic density distribution function in the form

$\rho(\mathbf{r})=\xi \Lambda^{-3} \cos \varphi(\mathbf{r})=$

$=\xi \Lambda^{-3}\left\{1+\cos \left(k_{x} x\right)+\cos \left(k_{y} y\right)+\cos \left(k_{z} z\right)\right\}$,

which corresponds to a cubic lattice with the wave vector $\mathbf{k}=\left(k_{x}, k_{y}, k_{z}\right)$. Supposing that there is one charged particle at every lattice site, and the lattice is isotropic, so that $k_{x}=k_{y}=k_{z}=2 \pi n^{1 / 3}$, where $n=N / V$ is the particle concentration, we obtain from the normalization condition that $\xi=\frac{N}{\Lambda^{-3} V}$. On the basis of the first integral for the field variable, we can conclude that the local entropy looks like

$S=S_{B}+N\left\{\frac{\pi^{2} \varkappa^{2}}{4 n r_{e}}-1\right\}$.

Then, introducing the coupling parameter $\Gamma_{e}=r_{e} n^{\frac{1}{3}}$, which is equal to the ratio between the Coulombic interaction and kinetic energies, we obtain the relation for the critical value of the coupling parameter,

$\Gamma_{e} \geq 4 \pi^{2} \varkappa^{2} n^{\frac{2}{3}} \equiv(2 \pi \varkappa L)^{2}$,

where $L$ is the lattice period. If this condition is satisfied, one may expect the formation of a crystalline structure.

Just such structures are observed in the case of dust plasma. As a structural parameter, we use the lattice constant $L$ (the distance between the particles normalized by the effective screening length). As a result, we obtain

$\Gamma_{e} \geq(2 \pi l)^{2}, \quad l \equiv \varkappa L$.

This relation gives values that are of the same order of magnitude as the result of the numerical simulation.

ISSN 2071-0194. Ukr. J. Phys. 2020. Vol. 65, No. 12 


\section{Self-Gravitating System}

Not less interesting are systems with purely attractive interaction. An example of such a situation is the behavior of a self-gravitating system. The solution of the saddle-point equation completely determines all thermodynamic parameters for the field $\psi$, which describes the general behavior of a self-gravitating system both in the cases of spatially homogeneous and inhomogeneous particle distributions. In other approaches, the dependence of the temperature on the spatial point is introduced through the polytropic dependence of the temperature on the particle concentration in the equation of state [19]. As a result, this dependence follows from the required thermodynamic state and can be found for various particle distributions.

Now, let us derive the saddle-point equation for the limiting values

$Q_{l}=\int D \varphi D \psi d \xi \exp \{S(\psi(\mathbf{r}), \xi(\mathbf{r}))\}$

of the local thermodynamic function

$$
\begin{aligned}
& S=\int\left[\frac{1}{2 r_{\psi}} \psi(\mathbf{r}) L_{\mathbf{r r}^{\prime}}^{\psi} \psi\left(\mathbf{r}^{\prime}\right)+\right. \\
& \left.+\xi(\mathbf{r}) \Lambda^{-3} \exp \sqrt{\beta(r)} \psi(\mathbf{r})\right] d \mathbf{r} .
\end{aligned}
$$

In the absence of a repulsive interaction $(\varphi=0)$, the equation for the field variable, $\delta S / \delta \psi=0$, brings about the expression

$$
\begin{aligned}
& \frac{1}{r_{m}} \Delta \psi(\mathbf{r})+\xi(\mathbf{r})\left(\frac{2 \pi m}{\hbar^{2} \beta(\mathbf{r})}\right)^{3 / 2} \times \\
& \times \sqrt{\beta(r)} \exp (\sqrt{\beta(r)} \psi(\mathbf{r}))=0,
\end{aligned}
$$

where the notation $r_{m} \equiv 4 \pi G m^{2}$ was introduced for the gravitational interaction length. The normalization condition can be written in the form

$\int \xi(\mathbf{r})\left(\frac{2 m}{\hbar^{2} \beta(\mathbf{r})}\right)^{3 / 2} \exp (\sqrt{\beta(r)} \psi(\mathbf{r})) d \mathbf{r}=N$,

and the conservation law for the energy of the system looks like

$$
\begin{aligned}
& \frac{1}{2} \int\left(\frac{2 \pi m}{\hbar^{2} \beta(\mathbf{r})}\right)^{3 / 2} \frac{\xi(\mathbf{r})}{\beta(\mathbf{r})}[3-\sqrt{\beta(r)} \psi(\mathbf{r})] \times \\
& \times \exp (\sqrt{\beta(r)} \psi(\mathbf{r})) d \mathbf{r}=E .
\end{aligned}
$$

In order to obtain a more information about the behavior of the self-gravitating system, we introduce new variables. The preservation of the particle number $\int \rho(\mathbf{r}) d \mathbf{r}=N$ gives us the following expression for the density function: 47

$\rho(\mathbf{r}) \equiv\left(\frac{2 \pi m}{\hbar^{2} \beta(\mathbf{r})}\right)^{3 / 2} \xi(\mathbf{r}) \exp (\sqrt{\beta(\mathbf{r})} \psi(\mathbf{r}))$,

which simplifies the previously obtained equation. In this case, the equation for the field variable is taken in the form

$\Delta \psi(\mathbf{r})+r_{m} \sqrt{\beta(\mathbf{r})} \rho(\mathbf{r})=0$.

If the temperature and the chemical activity are constant, this equation transforms into the well-known equation for the gravitational potential $\psi=\sqrt{\beta(\mathbf{r})} \psi$,

$\Delta \psi(\mathbf{r})=-4 \pi G m^{2} \beta \rho(\mathbf{r})$

The energy conservation equation looks now like

$\frac{1}{2} \int \frac{\rho(\mathbf{r})}{\beta(\mathbf{r})}[3-\sqrt{\beta(r)} \psi(\mathbf{r})] d \mathbf{r}=E$

The equations obtained in such a way cannot be solved in the general case. However, some variants of the self-gravitating system behavior under various external conditions can be analyzed. The chemical activity can be rewritten in terms of the chemical potential, $\xi(\mathbf{r})=\exp (\mu(\mathbf{r}) \beta(\mathbf{r}))$. By differentiating Eq. (50) with respect to the volume, we obtain the equation for the chemical potential,

$$
\begin{aligned}
& \frac{1}{2} \frac{\rho(\mathbf{r})}{\beta(\mathbf{r})}[3-\sqrt{\beta(r)} \psi(\mathbf{r})]=\frac{\delta E}{\delta V}= \\
& =\frac{\delta E}{\delta N} \frac{\delta N}{\delta V}=\mu(\mathbf{r}) \rho(\mathbf{r}) .
\end{aligned}
$$

From whence, we have

$\left.\mu(\mathbf{r}) \beta(\mathbf{r})=\frac{3}{2}-\frac{1}{2} \sqrt{\beta(r)} \psi(\mathbf{r})\right)$.

By introducing the reduced thermal de Broglie wavelength

$\Lambda(\mathbf{r})=\left(\frac{\hbar^{2} \beta(\mathbf{r})}{2 m e}\right)^{1 / 2}$

and the gravitation length

$R_{g}(\mathbf{r})=2 \pi G m^{2} \beta(\mathbf{r})$, 
all the equations and normalization conditions can be rewritten in terms of the density and temperature,

$\Delta\left(\frac{\ln \left(\Lambda^{3}(\mathbf{r}) \rho(\mathbf{r})\right)}{\sqrt{\beta(\mathbf{r})}}\right)+\frac{R_{g}(\mathbf{r})}{\sqrt{\beta(\mathbf{r})}} \rho(\mathbf{r})=0$.

The formula for the chemical potential is reduced to the expression

$\mu(\mathbf{r}) \beta(\mathbf{r})=\frac{3}{2}-\ln \left(\Lambda^{3}(\mathbf{r}) \rho(\mathbf{r})\right)$.

Thus, we can obtain the equation of state for a self-gravitating system, if we use the thermodynamic relation for the conservation of the energy $E$,

$P=-\frac{1}{\beta} \frac{\delta S}{\delta V}$

From the definition of the particle concentration, it follows that

$S=\int\left[-\rho(\mathbf{r}) \ln \left(\Lambda^{3}(\mathbf{r}) \rho(\mathbf{r})-\rho(\mathbf{r})\right] d \mathbf{r}\right.$,

so that the local equation of state can be written in the form

$P(\mathbf{r}) \beta(\mathbf{r})=\rho(\mathbf{r})\left(1-\ln \left(\Lambda^{3}(\mathbf{r}) \rho(\mathbf{r})\right)=\right.$

$=\rho(\mathbf{r})\left(\mu(\mathbf{r}) \beta(\mathbf{r})-\frac{1}{2}\right)$.

In the classical case, $\Lambda^{3}(\mathbf{r}) \rho(\mathbf{r}) \ll 1$ and $P \beta \equiv \rho$. At $\Lambda^{3}(\mathbf{r}) \rho(\mathbf{r})=1$, we obtain the equation of state for the ideal gas.

\section{Particle and Temperature}

\section{Distributions in a Self-Gravitating System}

\subsection{Homogeneous particle distribution}

First of all, let us consider the equilibrium state, when the parameters do not depend on the spatial coordinates. In this case, the energy and the total number of particles are fixed, and, furthermore, the temperature and the chemical potential do not vary in the space. Then the equation for the particle distribution

$\Delta \psi(\mathbf{r})+r_{m} \sqrt{\beta(\mathbf{r})} \rho(\mathbf{r})=0$

leads to a simple state with $\sqrt{\beta} \rho=0$, which can be realized, only if $T \rightarrow \infty$. Hence, the distribution of particles in a self-gravitating system can be homogeneous only at very high temperatures.

1068
Another interesting case takes place, if only the particle concentration depends on the coordinate, whereas the temperature is fixed. Then the equation for the density takes the form

$\Delta\left(\ln \Lambda^{3} \rho(\mathbf{r})\right)+R_{g} \rho(\mathbf{r})=0$

and can be transformed into

$\Delta(\ln \rho(\mathbf{r}))+R_{g} \rho(\mathbf{r})=0$.

Equation (60) has the exact solution $\rho(\mathbf{r})=2 /\left(R_{g} r^{2}\right)$, but the normalization condition is obeyed only in the case of a fixed volume with the size $R=$ $=\left(N G m^{2}\right) /(4 k T)$ and the fixed energy $E=N k T$. Then the change of the chemical potential in a confined volume is given by the expression

$\mu=k T\left(\frac{3}{2}-\frac{2 \Lambda^{3}}{4 k T R_{g} r^{2}}\right)$

By introducing a new function $f(\mathbf{r})=\ln \rho(\mathbf{r})$, Eq. (60) can be transformed into the Lane-Emden equation $[10,19]$

$\Delta f(\mathbf{r})+R_{g} \exp f(\mathbf{r})=0$,

which has the exact solution for the particle density

$\rho(\mathbf{r})=\frac{1}{\cosh ^{2}\left(r / R_{g}\right)}$

only in the one-dimensional case $[10,45,49]$. This equation does not agree with the results of the molecular dynamic simulation [49] and does not describe the general spatially inhomogeneous particle distribution in self-gravitating systems [10]. As follows from the equation obtained for a constant temperature, the homogeneous particle distribution is unstable, and the equation for the density perturbations $\rho(\mathbf{r})=$ $=\rho+\delta \rho(\mathbf{r})$ looks like

$\Delta \delta \rho(\mathbf{r})+R_{g} \rho \delta \rho(\mathbf{r})=0$,

which is the Helmholtz equation. The general solution of the wave equation is an unstable radial distribution $\delta \rho(\mathbf{r})=(\exp i k r) / r$ with the wave number $k=\sqrt{2 \pi G m^{2} \beta \rho}$. This means that the instability wavelength is half as large as the Jeans length. This is the statistical length of the instability for a particle distribution in the system. The concept of the Jeans gravitational instability is discussed in the framework of equilibrium statistics and the related kinetic theory [31].

ISSN 2071-0194. Ukr. J. Phys. 2020. Vol. 65, No. 12 


\subsection{Inhomogeneous particle and temperature distributions}

Now, let us consider the non-equilibrium description of a self-gravitating system and account for the probable spatially inhomogeneous distributions of particles and the temperature. Let us introduce the new variable $\phi=\Lambda^{3}(\mathbf{r}) \rho(\mathbf{r})$ and simplify the equation for the density. We get

$\Delta\left(\frac{\ln \phi}{\sqrt{\beta(\mathbf{r})}}\right)+\frac{R_{g}(\mathbf{r})}{\sqrt{\beta(\mathbf{r})} \Lambda^{3}(\mathbf{r})} \phi=0$.

In general, the exact solutions of this nonlinear equation are unknown, but we now propose a way to analyze this equation.

First of all, we can find the general solution of the problem. In the three-dimensional case, the action of the Laplace operator can be represented as follows:

$\Delta\left(\frac{\ln \phi}{\sqrt{\beta(\mathbf{r})}}\right)=\frac{1}{\sqrt{\beta}}\left(\frac{d^{2}}{d r^{2}}+\frac{2}{r} \frac{d}{d r}\right) \ln \phi-$

$-\frac{\ln \phi}{\sqrt{\beta^{3}}}\left(\frac{d^{2} \beta}{d r^{2}}+\frac{2}{r} \frac{d \beta}{d r}-\frac{3}{2 \beta}\left(\frac{d \beta}{d r}\right)^{2}\right)-$

$-\frac{1}{\sqrt{\beta^{3}}} \frac{d \ln \phi}{d r} \frac{d \beta}{d r}$.

A solution for the temperature can be sought in the form $\beta=\gamma^{3} r^{n}$. Then, if $n=2$, we obtain an equation only for $\phi$,

$\frac{d^{2} \ln \phi}{d r^{2}}+\frac{a_{m}}{B \gamma r} \phi=0$,

which can be rewritten in terms of the new variable $\bar{r}^{2}=r$ as follows:

$\frac{d}{d \bar{r}}\left(\frac{1}{\phi} \frac{d \phi}{d \bar{r}}\right)+\frac{4 a_{m}}{B \gamma} \phi=0$.

Let us multiply this equation by $(1 / \phi)(d \phi / d \bar{r})$ and calculate the first integral of the product,

$\left(\frac{1}{\phi} \frac{d \phi}{d \bar{r}}\right)^{2}+\frac{4 a_{m}}{B \gamma} \phi=\Delta$.

The exact solution of Eq. (67) is written in the form

$\phi=\frac{\Delta}{8 a_{m} / B \gamma \sinh ^{2} \sqrt{\Delta r / 4}}$.

ISSN 2071-0194. Ukr. J. Phys. 2020. Vol. 65, No. 12
With the help of formula (68), we obtain the exact solution for the inhomogeneous particle distribution,

$\rho(\mathbf{r})=\frac{\Delta}{8 a_{m} \gamma^{2} r^{3} \sinh ^{2} \sqrt{\Delta r / 4}}$.

This behavior correlates with the results obtained in works $[47,48]$, where the Boltzmann equation and the distribution function for a spherical isolated stellar system were used. The particle distribution is inhomogeneous for the size $R=1 / 4 \Delta$ and diverges at $r \rightarrow 0$ as $\rho(\mathbf{r})=1 / 2 a_{m} \gamma^{2} r^{4}$. The energy of the system is constant at that. However, we yet do not know the coefficients. To solve this problem, an approach described below is proposed.

If the particles are concentrated at short distances, and if their concentration is very high, then the quantum effect becomes crucial, and the classical approach is invalid. In this case, the relation between the critical temperature and the particle concentration is determined in a natural way,

$\Lambda^{3}(\mathbf{r}) \rho(\mathbf{r})=\left(\frac{\hbar^{2} \beta_{c}}{2 m e}\right)^{3 / 2} \rho_{c}=1$.

This relation together with the formula for the constant particle number, $4 \pi / 2 a_{m} R_{c}=N$, determine all necessary parameters, i.e. the critical distance $R_{c}=$ $=\hbar^{2} / m a_{m} N^{1 / 3}$, the coefficient $\gamma^{2}=(2 \pi m e) /\left(\hbar^{2} N^{2 / 3}\right)$, the critical temperature $\beta_{c}=\gamma^{2} R_{c}^{2}$, and the critical density $\rho_{c}=1 / 2 a_{m} R_{c}^{4}$. The energy of the system in this case is $E=\frac{3}{2} N k T$, i.e. it is equal to the energy of free particles!

Let us describe a system for which $\Lambda^{3}(\mathbf{r}) \rho(\mathbf{r})=$ $=\alpha=$ const $\ll 1$. In this case, only the temperature behavior can be determined, which is governed by the equation

$\Delta\left(\frac{1}{\sqrt{\beta(\mathbf{r})}}\right)+\frac{a_{m} e^{\alpha}}{B \ln \alpha} \frac{1}{\beta}=0$.

Similarly to the previous case, let us seek the solution of this equation in the form $\beta=\gamma^{-2} r^{-2 n}$. Then we find that it is obeyed for $n=-2$, i.e. the temperature varies as $k T=\gamma^{2} r^{-4}$, and the concentration as $\rho=A r^{-6}$. The normalization conditions for the constant particle number and energy are satisfied at that.

Hence, we have a solution that describes the spatial dependences of the concentration and the temperature. 


\section{Stationary States of Non-Equilibrium Systems}

Any macroscopic system, when being in a thermostat, approaches the equilibrium state during some relaxation time. In the equilibrium state, the properties of the system do not depend on the method of equilibrium establishment. However, the equilibrium state is realized only under certain idealized conditions. Therefore, actually, the properties of the system in the quasistationary (stationary) state may depend on the specific features of the interaction between the system and the thermostat, as well as on the parameters of the system $[14,21-54]$. The same is applicable to nonequilibrium systems, which can demonstrate, nevertheless, nevertheless, the equilibrium behavior.

Under certain conditions, the equilibrium state of the system may exist as a stationary state in which the energy exchange between the system and the environment is balanced. Such a state is a result of the balance between the direct action of the environment on the system and the process of energy dissipation taking place owing to the interaction of the system with the environment. It is easy to imagine a macroscopic system that can receive the energy from the environment but cannot return it all back, which depends on the parameters of the system. These are examples of such systems: hot electrons in a semiconductor system [3], a system of photons diffracting due to their scattering at inhomogeneities (the coefficient of photon diffraction depends on the photon frequency) [51, 55], a system of high-energy particles generated at collisions in an accelerator, and a system of ordinary Brownian particles with the friction coefficient depending on their velocity. All those systems are far from thermal equilibrium, and their new state is completely determined by the processes of energy exchange. Such a system may be characterized by a distribution function of its states that differs from the equilibrium distribution function, in particular, as the well-known distribution function of thermal states.

There is no clear definition of the distribution function for a non-equilibrium system that would taccount for possible states of the macroscopic system [3]. The standard method allows a non-equilibrium states to be considered as an equilibrium thermal state, if the deviations from this state are small. The imbalance manifests itself in this approach as small modifications in the equilibrium distribution function. An open system can exist very far from the equilibrium, but exhibiting, nevertheless, a stationary behavior.

In this section, we consider the problem of description of a non-equilibrium system and give one of the possible definitions for a new stationary state, which accounts for the energy absorption and the scattering processes induced by the interaction with the environment. Let us begin with the formulation of the statistical approach.

For an equilibrium state, the canonical distribution function in the phase space can be written in the form

$\rho(q, p) d \Gamma=\exp \left\{\frac{F-H(q, p)}{\Theta}\right\} d \Gamma$,

where $H(q, p)$ is the Hamiltonian, $d \Gamma=\prod_{i} d q_{i} d p_{i}$ is an element of the phase space, $\Theta=k T, T$ is the temperature, and $F$ is the free energy, which can be found from the normalization condition

$\int \exp \left\{\frac{F-H(q, p)}{\Theta}\right\} d \Gamma=1$.

As was shown in work [53], the phase space depends only on the energy $E$ of the system and the external parameters. Let us introduce the function

$\Sigma=\ln \frac{d \Gamma}{d E}$.

Then the canonical distribution function looks like

$\rho(E) d E=C \exp \left\{\frac{F-E}{\Theta}+\Sigma(E)\right\} d E$.

This relation describes the dependence of the distribution function on the energy of the macroscopic system [53]. The normalization condition can be written in this representation in the form

$\int C \exp \left\{\frac{F-E}{\Theta}+\Sigma(E)\right\} d E=1$,

which makes it possible to determine the normalization constant $C$. The latter accounts for the determinant transformation between the phase space and the energy variable. In order to distinguish the states that make a dominant contribution to the distribution function, we use the condition $d \Sigma / d E=1 / \Theta$, which defines the temperature of the system from the condition that the phase space depends only on the energy of the system. Using this definition and 
taking the basic principles of statistical mechanics into account [54], we arrive at the conclusion that $\Sigma=\ln d \Gamma / d E=S$ is equal to the entropy of the system.

It should be noted that the temperature describes the entropy dependence only on the energy, but not on other thermodynamic quantities. Another important conclusion is that the partition function can be calculated by integrating over the energy. The extremum of the distribution function is realized at $F=E-\theta S$, and any probable deviation from this condition makes a very small contribution to the macroscopic characteristics, similarly to the quantum-mechanical contribution to classical trajectories. This circumstance makes it possible to consider non-equilibrium systems as Brownian ones in the energy space [24]. On the basis of the Chapman-Kolmogorov equation for the distribution function of a macroscopic system in the energy space, the stationary states and fluctuation-dissipation relations for nonequilibrium systems can be obtained [24].

The energy, being regarded as a control quantity for a non-equilibrium system, can be a "slow" parameter describing the state of the system. In the absence of any other information about the non-equilibrium system, there is no preference among the states determined by the energy. Like in the equilibrium case, the non-equilibrium distribution function $\rho(E, t)$ can be determined as a function of the energy $E$ and the time. In the general case, it can be obtained from the basic kinetic equation. In terms of the energy, the basic kinetic equation for the non-equilibrium distribution function can be taken in the form

$$
\begin{aligned}
& \frac{\partial \rho(E, t)}{\partial t}=\int\left\{W\left(E, E^{\prime}\right) \rho\left(E^{\prime}, t\right)-\right. \\
& \left.-W\left(E^{\prime}, E\right) \rho(E, t)\right\} d E^{\prime},
\end{aligned}
$$

where $W\left(E, E^{\prime}\right)$ is the probability for the system to transit between the states with different energies. This basic kinetic equation is the balance equation for the probability of states. The energy representation of a non-equilibrium process is valid, only if this variable is canonical and performs averaging over the phase as the conjugate quantity. All solutions of the basic kinetic equation have a common property: as $t \rightarrow \infty$, they approach a stationary solution, which can be interpreted as the "equilibrium" state for the examined system. The stationary solution corresponds to the law of entropy growth [58].
In the case where the changes of the energy of the system are small, the basic kinematic equation transforms into the Fokker-Planck equation,

$\frac{\partial \rho(E, t)}{\partial t}=\frac{\partial}{\partial E} A(E) \rho(E, t)+$

$+\frac{1}{2} \frac{\partial^{2}}{\partial E^{2}} D(E) \rho(E, t)$.

The physical meaning of the coefficients $A(E)$ and $D(E)$ can be specified, if we return to the dynamic equation for the energy. In the general case, the dissipation equation can be written in the standard form of the Langevin equation

$\frac{d E}{d t}=f(E)+g(E) L(t)$.

This dissipation equation depends on external factors and initial conditions. The external influence mainly manifests itself in the changes of the energy of the system, which is either dissipated or absorbed. This process is described by the first term on the righthand side of Eq. (77), which describes the direct influence of the environment on the macroscopic system. This term can be obtained from the dynamics of any macroscopic system, if the direct interaction of this system with the environment is taken into account.

The changes in the energy of the system due to the stochastic influence of the environment is described by the second term on the right-hand side of Eq. (77). A random migration of the system is a result of the interaction between this system and the environment, the influence of which randomly modifies the energy of the system. Let us assume that the correlation between two fluctuation values at two different time moments, $\left\langle L(t) L\left(t^{\prime}\right)\right\rangle=\phi\left(t-t^{\prime}\right)$, can differ from zero only within a certain finite time interval. The symbol $\langle\ldots\rangle$ means the statistical averaging of the corresponding quantity. The function $\phi\left(t-t^{\prime}\right)$ must possess a sharp maximum near zero and satisfy the condition $\int \phi(\tau) d \tau=\sigma^{2}$ for the white noise [58]. A system that cannot restore its equilibrium state after rapid changes in the environment must adapt to new conditions. This process testifies to a possible dissipation of the energy, when the system contacts with the environment.

The energy representation of the general dissipation equation is also valid for an ordinary Brownian particle. The dynamics of Brownian particles can 
be described in terms of the velocity $v$ using the Langevin equation

$$
\frac{d v}{d t}=-\gamma v+F(t)
$$

where $\gamma$ is the friction coefficient, and $F(t)$ is a random force that describes some action on the particle in a liquid. In this case, the equalities $\langle F(t)\rangle=0$ and $\left\langle F(t) F\left(t^{\prime}\right)\right\rangle=\phi^{2} \delta\left(t-t^{\prime}\right)$ are true. They satisfy the white noise condition and describe the uncorrelated process of particle motion. For a Brownian particle, the energy equals $E=M v^{2} / 2$, so we can define the energy that varies as

$\frac{d E}{d t}=M v \frac{d v}{d t}=-2 \gamma E+\sqrt{2 M E} F(t)$,

which corresponds to Eq. (77) with $f(E)=-2 \gamma E$, $g(E)=\sqrt{E}$, and $L(t)=\sqrt{2 M} F(t)$. By solving the Langevin equation for the velocity, we can obtain [58] $\langle v(\infty)\rangle=\phi^{2} / 2 \gamma=k T / M$, as well as $\langle E\rangle=k T / 2$, where the thermostat temperature $T$ is introduced. By solving Eq. (79) in the approximation that does not take the correlation energy into account, we also obtain

$\sqrt{\langle E\rangle^{2}}=\frac{\sigma^{2}}{4 \gamma} \equiv \frac{\phi^{2}}{4 \gamma} 2 M=k T$,

which is completely responsible for the equilibrium state, as was in the previous result.

The descriptions of the process in terms of various variables are equivalent. The advantage of the energy representation consists in that it allows the "equilibrium" states of a non-equilibrium system to be determined. This approach is efficient in the cases where the direct effect of the interaction with the environment and the action of random forces can be determined, as well as because the energy is the slowest variable on which the relaxation of the system depends.

Two different approaches have been proposed to analyze random processes. If the coefficient $g(E)$ depends on the energy at the initial time moment, the equation for the non-equilibrium distribution function generates a Fokker-Planck equation in form (76). But if this coefficient depends on the energy before and after the transition, the diffusion equation can be written in the Stratonovich form,

$$
\frac{\partial \rho}{\partial t}=-\frac{\partial}{\partial E}(f(E) \rho)+\frac{\sigma^{2}}{2} \frac{\partial}{\partial E} g(E) \frac{\partial}{\partial E} g(E) \rho .
$$

In what follows, the latter (Stratonovich) representation will be used, because the both representations are interrelated $[58,59]$.

Equation (80) can be rewritten as a continuity equation,

$\frac{\partial \rho(E, t)}{\partial t}=\frac{\partial J(\rho(E, t))}{\partial E}$,

where

$J=-\left[f(E)-\frac{\sigma^{2}}{2} g(E) \frac{\partial}{\partial E} g(E)\right] \rho+\frac{\sigma^{2}}{2} g^{2}(E) \frac{\partial}{\partial E} \rho$.

By comparing (76) and (82), we find that

$A(E)=f(E)-\frac{\sigma^{2}}{2} g(E) \frac{\partial}{\partial E} g(E)$

and the diffusion coefficient

$D(E)=\frac{\sigma^{2}}{2} g^{2}(E)$.

The stationary solution of the Fokker-Planck equation with $J(\rho(E, t))=0$ can be presented in the form

$\rho_{s}(E)=A \exp \left\{\int_{E_{0}}^{E} \frac{2 f\left(E^{\prime}\right) d E^{\prime}}{\sigma^{2} g^{2}\left(E^{\prime}\right)}-\ln \frac{g(E)}{g\left(E_{0}\right)}\right\}$

or

$\rho_{s}(E)=A \exp \{-U(E)\}$,

where

$U(E)=\ln \frac{g(E)}{g\left(E_{0}\right)}-\int_{E_{0}}^{E} \frac{2 f\left(E^{\prime}\right) d E^{\prime}}{\sigma^{2} g^{2}\left(E^{\prime}\right)}$.

This distribution function has an extreme value at the energy that can be found as a solution of the equation

$U^{\prime}(\widetilde{E})=\frac{1}{D(E)}\left[D^{\prime}(E)-f(E)\right]$,

where the primed quantities mean their derivatives with respect to the energy. This equation is equivalent to the equation

$D^{\prime}(\widetilde{E})=f(\widetilde{E})$,

which establishes the relation between the dissipation (scattering) and the diffusion in the stationary case

ISSN 2071-0194. Ukr. J. Phys. 2020. Vol. 65, No. 12 
and completely determines a new "equilibrium" state of the system. The stationary non-equilibrium distribution function can be set equal to

$\rho_{s}(E)=\exp \{-U(\widetilde{E})\} \exp \left\{-U^{\prime \prime}(\widetilde{E}) E^{2}\right\}$,

where

$-U^{\prime \prime}(\widetilde{E})=\frac{1}{D(\widetilde{E})}\left[D^{\prime \prime}(\widetilde{E})-f^{\prime}(\widetilde{E})\right]$.

Taking relation (87) into account, we obtain $\rho_{s}(E)=$ $=\exp \{-U(\widetilde{E})\}$. In the general case, $f(E)$ is a nonlinear function of the state, and the diffusion coefficient depends on the energy, which makes it possible to reveal a lot of interesting situations, including noise-induced transitions into new stabler "equilibrium" states.

If $g(E)=1$, the stationary solution can be written in the form

$\rho(E)=A \exp \left\{\int_{E_{0}}^{E} \frac{f(E)}{\sigma^{2}} d E^{\prime}\right\}$,

where $E_{0}$ is the internal energy of the system. In the case of conservative system (if $f(E)=0$ ), the stationary solution does not depend on the energy. Note that the value $E=E_{0}$ is not only an internal limit, but also a stationary point in the absence of energy dissipation and stochastic diffusion.

In the case with the energy diffusion only (if $g(E)=$ $=1$ ), the equations for the non-equilibrium distribution function acquire the form of the simple diffusion equation, and their solutions

$\rho(E)=A \frac{1}{\sqrt{4 \pi \sigma^{2} t}} \exp \left\{-\frac{\left(E-E_{0}\right)^{2}}{4 \sigma^{2} t}\right\}$

describe the migration of the system over its energy. The mean-square deviation of the energy increases with the time according to the law $\left\langle\left(E-E_{0}\right)^{2}\right\rangle=$ $=2 \sigma^{2} t$. This solution corresponds to the evolution of a system that was described in the initial state by the equilibrium distribution function $\rho(E)=\delta\left(E-E_{0}\right)$.

If the diffusion coefficient depends on the energy, and the energy conservation law $f(E)=0$ holds true, then the stationary solution takes the form

$\rho_{s}(E)=A \exp \left\{-\ln \frac{g(E)}{g\left(E_{0}\right)}\right\}$, which corresponds to the canonical equilibrium distribution function

$\rho(E)=A \exp \left\{-\beta\left(E-E_{0}\right)\right\}$

only provided that the condition $g(E)=e^{\beta E}$ is satisfied, where $\beta$ is the inverse temperature of the medium. This is possible, if

$D(E)=\frac{\sigma^{2}}{2} g^{2}(E)=\frac{\sigma^{2}}{2} e^{2 \beta E}$.

The description in the energy space can be more illustrative, if the equilibrium states are described in the standard way. As an example, the stationary solution for an ordinary Brownian particle can be written in the form

$\rho_{s}(E)=A \exp \left\{-\frac{4 \gamma}{\sigma^{2}} E-\ln \sqrt{E}\right\} \equiv$

$\equiv A \frac{1}{\sqrt{E}} \exp \{-\beta E\}$

where the well-known ratio $2 \gamma / \sigma^{2}=\beta$ is applied. With the normalization condition $\int \rho_{s}(E) d E \equiv$ $\equiv \int \rho_{s}(p) d p$, the distribution function in the momentum space looks like

$\rho_{s}(p)=A \exp \left\{-\beta \frac{p^{2}}{2 M}\right\}=A \exp \left\{-\frac{M v^{2}}{2 k T}\right\}$.

If the dissipative function is represented in the form $f(E)=\alpha_{t} e^{\beta E}$, then the Langevin equation can be rewritten in another form,

$\frac{d e^{-\beta E(t)}}{d t}=-\beta \alpha_{t}$,

where the quantity $\alpha_{t}=\alpha+\xi_{t}$ consists of the constant part $\alpha$ and the part $\xi_{t}$ describing the white-noise effect of the environment [59].

Using the new variable $z=e^{-\beta E}$, the FokkerPlanck equation for the non-equilibrium distribution function can be written in the simpler form

$\frac{\partial \rho(z, t)}{\partial t}=\frac{\partial}{\partial z}[\alpha \beta \rho(z, t)]+\frac{\sigma^{2} \beta^{2}}{2} \frac{\partial^{2}}{\partial z^{2}} \rho(z, t)$.

Its stationary solution looks like

$\rho_{s}(z)=\exp \left\{\frac{2 \alpha}{\sigma^{2} \beta} z\right\}$. 
In the case $\beta E>1$, it can be represented as follows:

$\rho_{s}(E)=\exp \{-\beta E\} \quad$ at $\quad \frac{2 \alpha}{\sigma^{2} \beta}=1$.

In the white-noise case, the Fokker-Planck equation can be written in the form [59]

$\frac{\partial \rho(E, t)}{\partial t}=\frac{\partial}{\partial E}(\gamma E \rho(E, t))+\frac{\sigma^{2}}{2} \frac{\partial^{2}}{\partial E^{2}} E^{2} \rho(v, t)$.

The stationary solution of this equation is [59]

$\rho_{s}(E, t)=N E^{-\frac{1}{2}\left(\frac{2 \gamma}{\sigma^{2}}+1\right)}$

(it can be verified by substitution). This stationary solution coincides with the solution in the standard case, if the diffusion and friction coefficients do not depend on the energy. A similar result,

$\rho_{s}(v, t)=N v^{-\frac{2 \gamma}{\sigma^{2}}+1}$

can also be obtained in the velocity representation.

Let us imagine a situation where the energy of the system increases, but there is a mechanism that restricts its value. Such a process can be described with the help of the dissipative function $f(E)=\gamma E-E^{2}$, where the second term takes the indicated restriction into account. The corresponding Fokker-Planck equation looks like

$\frac{\partial \rho(E, t)}{\partial t}=\frac{\partial}{\partial E}\left[\left(\gamma E-E^{2}\right) \rho(E, t)\right]+$

$+\frac{\sigma^{2}}{2} \frac{\partial^{2}}{\partial E^{2}} E^{2} \rho(v, t)$

and its stationary solution is

$\rho_{s}(E, t)=N E^{-\frac{2 \gamma}{\sigma^{2}}+1} \exp \left\{-\frac{2}{\sigma^{2}} E\right\}$.

\section{Statistical Description of the Universe Evolution}

The proposed statistical approach makes it possible to attempt a description of the Universe birth and evolution on the basis of the general principles of statistical mechanics and quantum-mechanical theory. With this aim in view, we suggest the following assumptions.

i) The Universe is non-equilibrium a priori, but we may assume that the state of a subsystem that is a part of a large system is described by the local Gibbs distribution. The latter evolves following the way that provides the energy minimum and the entropy maximum for the selected subsystem.

ii) In the case of spontaneous generation of a scalar field in the vacuum, the ground-state energy of the "new" vacuum (i.e. the vacuum of the system "initial vacuum + scalar field") for fields of other characters must be lower than the ground-state energy of the "initial" vacuum. The self-consistent interaction of the scalar field with fluctuations of any other field ensures the energy conservation in the new state of the system. The calculation of the partition function for such a system testifies to the possibility of a phase transition in it from the state with the zero scalar field to a state with a finite spontaneously generated scalar field. As a result, owing to the spontaneous symmetry breaking, there appear real particles, which can be interpreted as a Big Bang.

iii) The Universe interacts with the fluctuating vacuum, and the Universe energy is not constant (the role of a thermostat for the Universe is played by the vacuum in which all possible fields fluctuate and interact with the Universe).

First of all, we assume that the ground state of the vacuum has the energy $E_{0}$. In so doing, we assume a possibility for all fields that can be generated in this vacuum to fluctuate. This assumption allows us to write the equation of state for the vacuum. From thermodynamic relations, it is possible to determine the pressure,

$P=-\frac{d E_{0}}{d V}=-\rho_{v}$

where $V$ is the volume, provided that the entropy is constant. For the pure vacuum, we may write

$E_{0}=\rho_{v} V$.

Here, we assume that the energy is an additive quantity with the density $\rho_{v}$. Equation (100) is the wellknown equation of state for the vacuum.

The Universe has been non-equilibrium since its birth. Therefore, in order to describe its evolution, it is worth introducing an additional quantity $t$ called "time" as an internal parameter. Then we may write

$\frac{d S}{d t}=\frac{1}{\Theta} \frac{d E}{d t}$

ISSN 2071-0194. Ukr. J. Phys. 2020. Vol. 65, No. 12 
From whence, it follows that the temporal change of the entropy is associated with the temporal change of the energy. Since $d S / d t>0$, it follows that, in the course of a relaxation to the equilibrium state, we have $d E / d t>0$, i.e. the entropy increases together with the energy. In thermodynamics, heat is the energy distributed among the degrees of freedom that are not macroscopically observable. Therefore, when the vacuum state changes, let us suppose that $d Q / d t=d E / d t>0$, so that the heating is possible only at the relaxation to the equilibrium state.

The speculations and relations presented above are well known. Let us apply them in order to propose another possible explanation of the Universe evolution. As was mentioned above, according to standard cosmological models, an instability of the fundamental scalar field was the main origin of the formation of such a Universe state that resulted in the Big Bang. Therefore, let us first assume that there appeared a non-zero scalar field in the "initial" vacuum owing to a phase transition to its new state. This means that, after its appearance, the "new" vacuum with any other allowable field will differ from the "primary" vacuum, because the scalar field already exists in the former. The scalar field lowers the energy of the "new" vacuum with respect to that of the "primary" vacuum, and the ground-state energy in the "new" vacuum equals

$E=E_{0}-\frac{\mu^{2}}{2} \varphi^{2}$,

where the second term is the scalar field energy, and the coefficient $\mu^{2}$ describes the relation between the new field and the "primary" vacuum, i.e. the selfconsistent interaction of the new field with the fluctuations of all other fields that can exist in the "primary" vacuum.

Two remarks are to be made. First, the appearance of a scalar field lowers the energy of the initial vacuum state. Second, the coupling coefficient $\mu^{2}$ is now positive by definition and, therefore, does not require further explanations, which are usually made in the standard approach. The contribution of such interaction to the partition function of the Gibbs distribution can be described in the standard way:

$$
\begin{aligned}
& Z \sim \int D \varphi \int D \xi \times \\
& \times \exp \frac{1}{\Theta}\left\{-E_{0}+\frac{1}{2} \mu^{2} \varphi^{2}+\xi \varphi^{2}-\frac{\xi^{2}}{2 \sigma^{2}}\right\} .
\end{aligned}
$$

Here, we used the representation $\mu^{2}=\mu^{2}+\xi$ by assuming that the coupling coefficient consists of its average value and a fluctuation associated with the non-linear interaction of the field $\varphi$ with the fluctuations of the fields of different nature. We also supposed that the mean-square value of the fluctuation $\xi$ is equal to $\sigma^{2}$. Integrating with respect to the fields $\xi$, we obtain

$Z \sim \int D \varphi \exp \frac{1}{\Theta}\left\{-E_{0}+\frac{1}{2} \mu^{2} \varphi^{2}+-\frac{\sigma^{2} \varphi^{4}}{4}\right\}$.

So, we have a system with the effective energy (averaged over the fluctuations of another field with which the scalar field interacts)

$E=E_{0}+V(\varphi)=E_{0}-\frac{1}{2} \mu^{2} \varphi^{2}+\frac{\sigma^{2} \varphi^{4}}{4}$

where

$V(\varphi)=-\frac{1}{2}\left\langle\mu^{2}\right\rangle \varphi^{2}+\frac{\sigma^{2} \varphi^{4}}{4}$

is the well-known expression for the energy of the fundamental scalar field. The total effective energy of the "new" vacuum with the fundamental scalar field can be written in the form

$E=E_{0}-\frac{\left\langle\mu^{2}\right\rangle^{2}}{4 \sigma^{2}}+\frac{\sigma^{2}}{4}\left(\varphi^{2}-\frac{\left\langle\mu^{2}\right\rangle}{\sigma^{2}}\right)^{2}$.

From whence, it follows that $E=E_{0}$ in the absence of a scalar field $(\varphi=0)$, whereas, at

$\varphi^{2}=\frac{\left\langle\mu^{2}\right\rangle}{\sigma^{2}}$

the effective energy of the ground state of the "new" vacuum equals

$E=E_{0}-\frac{\left\langle\mu^{2}\right\rangle^{2}}{4 \sigma^{2}}$

From Eq. (106), one can see that the energy of the vacuum with the scalar field is lower than the energy of the "primary" vacuum and can equal zero, if

$E_{0}=\frac{\left\langle\mu^{2}\right\rangle^{2}}{4 \sigma^{2}}$

From the obtained formulas, it follows that the system may undergo a phase transition giving rise to the formation of a new ground state of the vacuum with energy (106), which can be equal to zero, if condition 
(107) is satisfied. The scalar field can be arbitrary large at that.

For the further consideration, let us suppose that both the statistical distribution in and the evolution of the Universe can be described by a distribution function that depends only on the energy. An example of how this idea can be applied to describe the features in the statistical distribution was given in the previous section. Concerning the description of the evolution for non-equilibrium distributions (with our Universe being non-equilibrium a priori), the assumption that the system evolves in the energy space similarly to the Brownian motion of the system in the energy-state space seems to be quite natural. The answer to question "What can serve as a thermostat whose interaction with the Universe is the source of system's random motion?" is as follows: it is the vacuum in which, as was noted above, all physical fields fluctuate, those fluctuations interact with the fundamental scalar field and, in such a way, affect even the ground state of the vacuum. In other words, it is the fluctuations of physical fields (except those for the selected fundamental scalar field) that play the role of a random source in the Langevin equations. This means that we can use the Fokker-Planck equation in the Stratonovich form (80) as the main equation to describe the evolution of the Universe.

Furthermore, if we also assume that the dissipative function $f(E)$ depends non-linearly on the energy, and a noise-induced transition to a new stabler stationary state can be implemented in the system, then the proposed formalism can be applied to describe Universe's formation. For this purpose, we have to formulate an equation that would describe the energy dissipation in the system with a scalar field. In the general form, such an equation looks like

$\frac{d E}{d t}=\frac{d E}{d \varphi} \frac{d \varphi}{d t}$

and the dynamic equation for the scalar field can be written in the standard form [54]

$\frac{d \varphi}{d t}=-\gamma \frac{d F(\varphi)}{d \varphi}$

Here, $\gamma$ is the friction coefficient describing the dissipation of the fundamental scalar field, and $F(\varphi)$ is the free energy. From the theory of phase transitions for the fundamental field [42], it is known that
$F(\varphi \Theta)=V(\varphi, 0)$ at the zero temperature so that Eq. (109) acquires a form that is well known in the standard cosmology. By setting

$\frac{d E}{d \varphi}=\frac{d V(\varphi)}{d \varphi}$

we obtain

$\frac{d E}{d t}=-\gamma\left(\frac{d V(\varphi)}{d \varphi}\right)^{2}$

In other words, we have obtained the generalized Langevin equation

$\frac{d E}{d t}=f(E)$

with

$f(E)=-\gamma\left(\frac{d V(\varphi)}{d \varphi}\right)^{2}$

In the simplest case,

$V(\varphi)=-\frac{1}{2} \mu^{2} \varphi^{2}$

and

$f(E)=-2 \gamma \mu^{2} E$.

In view of the assumption made above that $\mu^{2}=$ $=\mu^{2}+\xi$, as well as the Langevin equation (111), we arrive at the expression $g(E)=2 \gamma E$, which yields the following stationary solution:

$\rho(E, t)=N(\gamma E)^{-\frac{\left\langle\mu^{2}\right\rangle}{\gamma \sigma^{2}}+1}$.

The same solution is obtained for the Fokker-Planck equation [59],

$\rho(E, t)=N\left(\frac{E}{E_{0}}\right)^{-\frac{\left\langle\mu^{2}\right\rangle}{\gamma \sigma^{2}}+1}$.

From the presented results, it follows that one of the possible Universe states is a state characterized by a scalar order parameter and possessing the possibility to form a "bubble" of a new state with the energy $E=E_{0}-\mu^{4} / 4 \sigma^{2}$. The entropy of the ground state with the vacuum energy $E_{0}$ is minimum and increases with the formation of a bubble of the new phase. The starting point in all cosmological models is the Big Bang that generates a heated Universe. Our

ISSN 2071-0194. Ukr. J. Phys. 2020. Vol. 65, No. 12 
approach can shift the starting point. We can start the consideration from the vacuum state with field fluctuations of various nature. At the beginning of the "time", when an additional scalar field appears, the number of possible states increases and the energy of the new vacuum state decreases owing to the formation of growing bubbles of the new state. In particular, at the critical temperature $\Theta_{c}=2 \mu / \sigma$ [42], the symmetry in the behavior of the fundamental scalar field is restored, and we have the well-known behavior of the Universe, but with a principal difference. The initial vacuum remains restored incompletely, and we can observe the expansion of the Universe with acceleration. As was noted above, this process depends on the dispersion of field fluctuations of various nature. But if a new-phase "bubble" has been formed, not all possible fluctuations can affect the processes running in the Universe. This is known as the fluctuation-induced condensation effect [38]. In this case, the acceleration never terminates.

To calculate the transient probabilities, the interaction with a multiplicative noise should be considered from the viewpoint of possible changes in both the dispersion of fluctuations and the scalar field potential. In so doing, the non-linearity of the scalar field potential and the field fluctuations of various nature should be taken into consideration. Such fluctuations can modify the potential minimum and determine an alternative way for Universe's formation. For example, the Universe state can be described in this case as $\varphi=0$, but with a non-zero Hubble constant. Thus, a new model has been proposed to describe the nonequilibrium Universe, which makes it possible to determine new stationary states.

\section{Conclusions}

In many cases, the systems with long-range interactions are non-equilibrium. Before relaxing to the thermodynamic equilibrium, the systems with longrange interaction turn out in non-equilibrium quasistationary states whose lifetime diverges, if the number of particles tends to infinity. Therefore, in the thermodynamic limit, the interacting systems cannot relax to an equilibrium. The modern statistical description of non-equilibrium states considers only probable structures in a self-gravitating system, but does not describe metastable states and says nothing about the time scales of the kinetic theory. In this work, a new approach has been proposed which uses the non-equilibrium statistical operator involving the inhomogeneous particle and temperature distributions.

To find the dominant contribution to the distribution function, the saddle-point method is used, which made it possible to obtain all thermodynamic parameters of the system and, thus, shed light on the problem of self-gravitation in systems with the inhomogeneous particle and temperature distributions. Probable features in the behavior of self-gravitating system under various conditions are predicted. The equation of state for a self-gravitating system is derived. The new length of a statistical instability and the parameters of spatially inhomogeneous particle and temperature distributions are found for real gravitational systems.

An approach allowing the quantitative prediction of a particle distribution in a self-gravitating system is suggested. The formation of spatially inhomogeneous particle distributions accompanied by changes in the temperature is described for the first time. The statistical description of the system is used to interpret the behavior of gravitating particles in the case of arbitrary spatially inhomogeneous particle distributions. Starting from general initial conditions, the system evolves to one of plenty of macroscopically stationary states.

Thus, we can solve a difficult problem dealing with the statistical description of systems with longrange interaction. In addition, this method can be used to develop further the physics of self-gravitating and similar systems in the non-equilibrium state. The approach to describe non-equilibrium states of a macroscopic system is proposed. On the basis of the Fokker-Planck equation for the non-equilibrium distribution function of a macroscopic system, stationary solutions are obtained. The proposed approach allows one to analyze the Brownian dynamics between various states of the system that are induced by the energy dissipation and the influence of the environment, both factors depending on the energy of the system. It is shown that the equilibrium state of the system, when the energy exchange between the system and the environment is in balance, can be regarded as a stationary state. Such state is a result of the balance between the direct action of the environment on the system and the dissipation process induced by the interaction with the environment. 
This work was carried out in the framework of the priority theme of the Department of Physics and Astronomy of the National Academy of Sciences of Ukraine "Mathematical models of non-equilibrium processes in open systems" (No. 0120U100857).

1. W. Thirring. Systems with negative specific heat. Z. Phys. 235, 339 (1970).

2. P.-H. Chavanis, C. Rosier, C. Sire. Thermodynamics of self-gravitating systems. Phys. Rev. E 66, 036105 (2002).

3. D.N. Zubarev. Non-Equilibrium Statistical Thermodynamics (Consultants Bureau, 1974).

4. V. Laliena. On the thermodynamical limit of self-gravitating systems. Nucl. Phys. B 668, 403 (2003).

5. R. Pakter, B. Marcos, Y. Levin. Symmetry breaking in $d$ dimensional self-gravitating systems. Phys. Rev. Lett. 111, 230603 (2013).

6. F.P.C. Benetti, A.C. Ribeiro-Teixeira, R. Pakter, Y. Levin. Nonequilibrium stationary states of $3 \mathrm{D}$ self-gravitating systems. Phys. Rev. Lett. 113, 100602 (2014).

7. R. Baxter. Exactly Solved Models in Statistical Mechanics (Academic Press, 1980).

8. D. Ruelle. Statistical Mechanics (Rigorous Results) (Benjamin, 1969).

9. Y.D. Bilotsky, B.I. Lev. Clustering in condensed media. Teor. Mat. Fiz. 60, 711 (1984) (in Russian).

10. B.I. Lev, A.Ya. Zhugaevych. Statistical description of model systems of interacting particles and phase transitions accompanied by cluster formation. Phys. Rev. E. 57, 6460 (1998).

11. B.I. Lev. Nonequilibrium self-gravitating system. Int. J. Mod. Phys. B 25, 2237 (2011).

12. H. Kleinert. Gauge Field in Condensed Matter (Word Scientific, 1989).

13. R.L. Stratonovich. On a method of calculating quantum distribution functions. Sov. Phys. Dokl. 2, 416 (1958).

14. J. Hubbard. Calculation of partition functions. Phys. Rev. Lett. 3, 77 (1958).

15. K.V. Grigorishin, B.I. Lev. Cluster formation in the system of interacting Bose particles. Phys. Rev. E 71, 066105 (2005).

16. T. Padmanabhan. Statistical mechanics of gravitating system. Phys. Rep. 188, 285 (1990).

17. P.-H. Chavanis. Phase transitions in self-gravitating systems.Int. J. Mod. Phys. B 20, 3113 (2006).

18. S. Chandrasekhar. An Introduction to the Study of Stellar Structure (Dover, 1942).

19. C. Sire, P.-H. Chavanis. Thermodynamics and collapse of self-gravitating Brownian particles in $D$ dimensions. Phys. Rev. E 66, 046133 (2002).

20. E.B. Aronson, C.J. Hansen. Thermal equilibrium states of a classical system with gravitation. Astrophys. J. 117, 145 (1972).

21. K. Huang. Statistical Mechanics (Wiley, 1963) [ISBN: 9780-471-81518-1].
22. A. Isihara. Statistical Physics (Academic Press, 1971).

23. S. Shaikh, A. Khan. Instability of thermally conducting self-gravitating systems. J. Mod. Phys. 1, 77 (2010).

24. B.I. Lev. Brownian system in energy space. Eur. Phys. J. Spec. Topics 216, 37 (2013).

25. H.-J. de Vega, N. Sánchez. The statistical mechanics of the self-gravitating gas: equation of state and fractal dimension. Phys. Lett. B 490, 180 (2000).

26. H.-J. de Vega, N. Sánchez. Statistical mechanics of the self-gravitating gas: I. Thermodynamic limit and phase diagrams. Nucl. Phys. B 625, 409 (2002).

27. H.-J. de Vega, N. Sánchez. Statistical mechanics of the selfgravitating gas: II. Local physical magnitudes and fractal structures. Nucl. Phys. B 625, 460 (2002).

28. W.C. Saslow. Gravitational Physics of Stellar and Galactic Systems (Cambridge Univ. Press, 1987).

29. D. Lynden-Bell, R. Wood. The gravo-thermal catastrophe in isothermal spheres and the onset of red-giant structure for stellar systems. Mon. Not. R. Astron. Soc. 138, 495 (1968).

30. H.J. de Vega, N. Sánchez, F. Combes. Fractal dimensions and scaling laws in the interstellar medium: A new field theory approach. Phys. Rev. D 54, 6008 (1996).

31. J.A.S. Lima, R. Silva, J. Santos. Jeans' gravitational instability and nonextensive kinetic theory. Astron. Astrophys. 396, 309 (2002).

32. A.P. Boss. Giant planet formation by gravitational instability. Science 276, 1836 (1997).

33. S.M. Fall. Gravitational instability theory of galaxy formation and clustering: Some recent developments. Ann. NY Acad. Sci. 336, 172 (2006).

34. H. Kleinert. Collective quantum fields. Fortschr. Phys. 26, 565 (1979).

35. L.N. Lipatov. Divergence of the perturbation-theory series and the quasi-classical theory. JETP (Sov) 72, 412 (1977).

36. S. Edward, A. Lenard. Exact statistical mechanics of a onedimensional system with Coulomb forces. II. The method of functional integration. J. Math. Phys. 3, 778 (1962).

37. B.I. Lev, A.G. Zagorodny. Statistical description of Coulomb-like systems. Phys. Rev. E 84, 061115 (2011).

38. B.I. Lev. Statistical induced dynamic of self-gravitating system. J. Mod. Phys. 10, 687 (2019).

39. B.I. Lev. Statistical derivation of the fundamental scalar field. J. Mod. Phys. 9, 2223 (2018).

40. B.I. Lev, S.B. Lev. Statistical description of nonequilibrium self-gravitating systems. Eur. Phys. J. B 9, 3 (2017).

41. S. Samuel. Grand partition function in field theory with applications to sine-Gordon field theory. Phys. Rev. D 18, 1916 (1978).

42. A.D. Linde. Elementary Particle Physics and Inflationary Cosmology (Harwood Academic, 1990).

43. A.D. Linde. Phase transitions in gauge theories and cosmology. Rep. Prog. Phys. 42, 389 (1979).

44. S. Coleman. Fate of the false vacuum: Semiclassical theory. Phys. Rev. D 15, 2929 (1977); 16, 1762 (1977).

ISSN 2071-0194. Ukr. J. Phys. 2020. Vol. 65, No. 12 
45. G. Rybicki. Exact statistical mechanics of a onedimensional self-gravitating system. Astrophys. Space Sci. 14, 56 (1971).

46. K.R. Yawn, B.N. Miller. Incomplete relaxation in a twomass one-dimensional self-gravitating system. Phys. Rev. E 68, 056120 (2003).

47. R. Michie. On the distribution of high energy stars in spherical stellar systems. Not. R. Astron. Soc. 125, 127 (1962).

48. W. Jaffe. A simple model for the distribution of light in spherical galaxies. Not. R. Astron. Soc. 202, 995 (1983).

49. Y. Levin, R. Pakter, F.B. Rizzato, T.N. Teles, F.P.C. Benetti. Nonequilibrium statistical mechanics of systems with long-range interactions. Phys. Rep. 535, 1 (2014).

50. J.W. Cahn. On spinodal decomposition. Acta Metallurgica 9, 795 (1988).

51. C.W. Gardiner, P. Zoller. Quantum Noise (Springer, 2000) [ISBN: 978-3-540-22301-6].

52. R. Balescu. Equilibrium and Nonequilibrium Statistical Mechanics (Wiley, 1978).

53. J.W. Gibbs. Elementary Principles in Statistical Mechanics, Developed with Especial Reference to the Rational Foundation of Thermodynamics (Scribner's Sons, 1902).

54. L.D. Landau, E.M. Lifshitz. Statistical Physics (Pergamon, 1978).

55. D.F. Wells, G.J. Milburn. Quantum Optics (Springer, 2001).

56. P. Colet, F. De Pasquele, M. San Miguel. Relaxation in the subcritical pitchfork bifurcation: From critical to Gaussian scaling. Phys. Rev. A 43, 5296 (1991).

57. F.D.M. Haldane. "Fractional statistics" in arbitrary dimensions: A generalization of the Pauli principle. Phys. Rev. Lett. 67, 937 (1991).

58. N.G. van Kampen. Stochastic Processes in Physics and Chemistry (North-Holland, 1990).
59. W. Horsthemke, R. Lefever. Noise-Induced Phase Transitions. Theory, Applications in Physics, Chemistry and Biology (Springer, 1984).

60. A. Albrecht, P.J. Steinhard. Cosmology for grand unified theories with radiatively induced symmetry breaking. Phys. Rev. Lett. 48, 1220 (1982).

61. B.I. Lev, H. Yokoyama. Selection of states and fluctuation under the first order phase transitions. Int. J. Mod. Phys. $B$ 17, 4913 (2003).

Received 21.05.20

Translated from Ukrainian by O.I. Voitenko

\section{Б.І. Лев, А.Г. Загородній}

СТАТИСТИЧНЕ ОПИСАННЯ

НЕРІВНОВАЖНИХ БАГАТОЧАСТИНКОВИХ СИСТЕМ

$\mathrm{P}$ е $з$ ю м е

Системи частинок, що взаємодіють, в багатьох випадках є нерівноважними. В даному огляді представлено новий підхід, заснований на застосуванні нерівноважного статистичного оператора, який дає змогу врахувати неоднорідний розподіл частинок і температури. Такий метод використовує процедуру сідлової точки для знаходження основних внесків у статистичну суму і надає можливості отримати всі термодинамічні параметри систем. Передбачено можливі особливості поведінки взаємодійних систем, таких як гравітівні системи, системи з кулонівським відштовхуванням тощо для різних термодинамічних умов. Запропоновано новий підхід для описування нерівноважних систем в енергетичному просторі, що є розширенням підходу Гібса для макроскопічних систем за нерівноважних умов. Цей підхід уможливлює описати стаціонарні стани нерівноважних систем та їхню динаміку. 